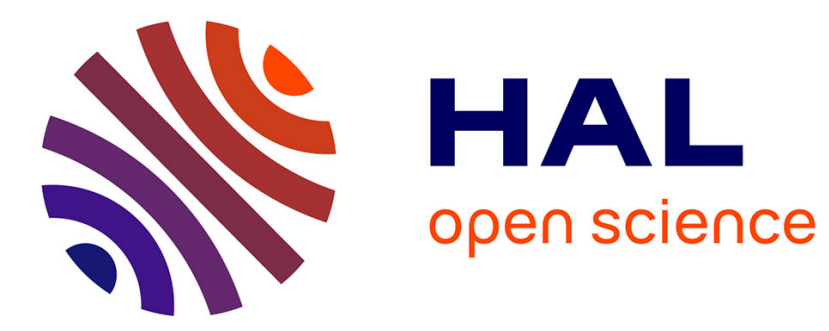

\title{
Large-Eddy simulation of turbulent pipe flow of power-law fluids
}

P. S. Gnambode, P. Orlandi, Meryem Ould-Rouiss, Xavier Nicolas

\section{To cite this version:}

P. S. Gnambode, P. Orlandi, Meryem Ould-Rouiss, Xavier Nicolas. Large-Eddy simulation of turbulent pipe flow of power-law fluids. International Journal of Heat and Fluid Flow, 2015, 54, pp.196-210. 10.1016/j.ijheatfluidflow.2015.05.004 . hal-01163467

\section{HAL Id: hal-01163467 \\ https://hal.science/hal-01163467}

Submitted on 12 Jun 2015

HAL is a multi-disciplinary open access archive for the deposit and dissemination of scientific research documents, whether they are published or not. The documents may come from teaching and research institutions in France or abroad, or from public or private research centers.
L'archive ouverte pluridisciplinaire HAL, est destinée au dépôt et à la diffusion de documents scientifiques de niveau recherche, publiés ou non, émanant des établissements d'enseignement et de recherche français ou étrangers, des laboratoires publics ou privés. 


\title{
Large-Eddy simulation of turbulent pipe flow of power-law fluids.
}

\author{
P.S. Gnambode, P. Orlandi ${ }^{1}$, M. Ould-Rouiss, ${ }^{*}$ and X. Nicolas \\ Université Paris-Est, Laboratoire Modélisation et Simulation Multi Echelle, \\ MSME, UMR 8208 CNRS, 5 bd Descartes, 77454 Marne-la-Vallée, France \\ 1 Dipartimento di Ingegneria Meccanica e Aerospaziale, \\ Università La Sapienza, Rome, Italy
}

(Dated: March 27, 2015)

\begin{abstract}
Fully developed turbulent flows of power-law fluids in a cylindrical stationary pipe are investigated numerically by the use of large eddy simulation (LES) for various power law index $(0.5 \leq n \leq 1.4)$ at different Reynolds numbers $\left(4000 \leq R e_{s} \leq 12000\right)$. To validate the present computations, the predictions are compared to the results reported in the archival literature for laminar and turbulent flows. The LES predictions agree reasonably favourably with the findings of the literature. The log-region of the mean axial velocity profile expands with increasing $R e_{s}$ and decreasing power-law index $n$. The predicted friction factor for $n \leq 1$ at $R e_{s}=4000$ is slightly overestimated in comparison with Dodge and Metzner correlation, and is better interpolated by Gomes correlation. With increasing $n$ the apparent viscosity increases close to the wall and decreases for $y^{+}>30$. This implies that the turbulent fluctuations develop and are more intense further from the wall when $n>1$ and closer to the wall when $n<1$. The influence of $R e_{s}$ and $n$ on the higher-order statistics (skewness and flatness) is analyzed. Visualizations of the instantaneous filtered velocity fields exhibit turbulent patterns which develop more as $n$ increases.
\end{abstract}

\section{Keywords}

Large eddy simulation (LES); power-law fluid; pipe flow.

* corresponding author : ould@univ-mlv.fr

Tel : 01.60.95.73.09

Fax : 01.60.95.72.94 


\section{Nomenclature}

$C_{d} \quad$ coefficient of the dynamic model

$D \quad$ pipe diameter $(m)$

$f \quad$ mean friction factor, $f=2 \tau_{w} /\left(\rho U_{b}^{2}\right)$

$f_{D M} \quad$ Dodge and Metzner (1959) correlation for friction factor

$f_{G} \quad$ Gomes (1987) correlation for friction factor

$F\left(v_{i}^{\prime}\right) \quad$ flatness factor, $F\left(v_{i}^{\prime}\right)=\frac{\left\langle v_{i}^{\prime 4}\right\rangle}{\left\langle v_{i}^{\prime 2}\right\rangle^{2}}$

K consistency index $\left(P a . s^{n}\right)$

$L_{z} \quad$ length of the computational domain $(m)$

$n \quad$ power law index

$q_{i} \quad$ generic notation for $q_{r}, q_{\theta}$ and $q_{z}$

$q_{r}, q_{\theta}, q_{z} \quad$ variables $q_{r}=r v_{r}, q_{\theta}=r v_{\theta}, q_{z}=v_{z}$

$R e_{b} \quad$ Reynolds number based on bulk velocity, $R e_{b}=\frac{U_{b} D}{\nu}$

$R e_{c r} \quad$ critical Reynolds number between laminar and turbulent flows, $R e_{c r}=2100 \frac{(4 n+2)(5 n+3)}{3(3 n+1)^{2}}$

$R e_{M R} \quad$ Metzner-Reed Reynolds number $R e_{M R}=\frac{8 \rho U_{b}^{2-n} D^{n}}{K(6+2 / n)^{n}}$

$R e_{s} \quad$ simulation Reynolds number, $R e_{s}=\frac{\rho U_{c L}^{2-n} R^{n}}{K}$

$R e_{w} \quad$ generalized Reynolds number, $R e_{w}=\frac{\rho U_{\tau} D}{\eta_{w}}$

$r \quad$ dimensionless coordinate in the radial direction scaled by the pipe radius

$R \quad$ pipe radius $(m)$

$S_{i j} \quad$ strain rate tensor

$S\left(v_{i}^{\prime}\right) \quad$ skewness factor, $S\left(v_{i}^{\prime}\right)=\frac{\left\langle v_{i}^{\prime 3}\right\rangle}{\left\langle v_{i}^{\prime 2}\right\rangle^{\frac{3}{2}}}$

$u_{i} \quad$ generic notation for the dimensionless velocity components $v_{r}, v_{\theta}$ and $v_{z}$

$U_{b} \quad$ bulk velocity $(\mathrm{m} / \mathrm{s})$

$U_{c L} \quad$ centerline axial velocity. For analytical laminar profile, $U_{c L}=\frac{(3 n+1) U_{b}}{n+1}$

$U^{+} \quad$ mean axial velocity in wall units, $U^{+}=U / U_{\tau}$

$U_{\tau} \quad$ friction velocity, $U_{\tau}=\left(\tau_{w} / \rho\right)^{1 / 2}$

$v_{r}, v_{z}, v_{\theta} \quad$ dimensionless radial, axial and azimuthal velocity components

$y^{+} \quad$ distance from the wall in wall units, $y^{+}=(1-r) U_{\tau} / \nu$

$z \quad$ dimensionless coordinate in the axial direction scaled by the pipe radius

Greek symbols

$\eta_{w}$

mean apparent viscosity at the wall $\left(\mathrm{m}^{2} \mathrm{~s}^{-1}\right)$. 
$\eta$

$\eta_{d, w}$

$\dot{\gamma}$

$\dot{\gamma}_{d, w}$

$\theta$

$\nu_{t}$

$\rho$

$\tau_{i j}$

$\tau_{w}$

apparent viscosity, $\eta=K \dot{\gamma}^{n-1}$

dimensionless apparent viscosity at the wall, $\eta_{w} /\left(\rho U_{c L} R\right)$

shear rate, $\dot{\gamma}=\left(2 S_{i j} S_{i j}\right)^{1 / 2}$

dimensionless shear rate at the wall, $\dot{\gamma}_{d, w}=\gamma_{w} R / U_{c L}$

dimensionless angular coordinate in the circumferential direction

turbulent viscosity

density $\left(\mathrm{kg} / \mathrm{m}^{3}\right)$

subgrid stress tensor, $\bar{\tau}_{i j}=-2 \nu_{t} \bar{S}_{i j}$

mean averaged fully-established wall shear stress, $\tau_{w}=\frac{D}{4} \frac{\partial p}{\partial z}$

Superscripts

$<()>$.$\quad statistically averaged$

$(.)^{+} \quad$ normalized by $u_{\tau}$ or $\eta_{w}$

(.) filtered variable

$(.)^{\prime} \quad$ fluctuation component

Subscripts

$\begin{array}{ll}c & \text { centerline } \\ L & \text { laminar } \\ w & \text { wall }\end{array}$




\section{Introduction}

The turbulent flows of non-Newtonian fluids are of importance in mechanical and engineering fields. They are encountered in a variety of engineering applications, e.g. drilling hydraulics, sewage transport, processing of mineral oil and polymer products, blood flow in arteries, and applications involving relatively high heat transfer rates. While the turbulence theory, the mathematical models and the numerical methods are well-advanced for Newtonian fluids, those for non-Newtonian fluids are not as developed. Some attempts have been made to explore the effect of the power-law index and Reynolds number on the velocity distribution and turbulence statistics. Computational models for non-Newtonian fluids can help to bridge the gap in the existing literature, and can contribute to developing the general theories of the turbulent flows of non-Newtonian fluids.

Malin (1997) used a modified $k-\epsilon$ model (a low Reynolds number $k-\epsilon$ model extended to power law fluids) to calculate the frictional resistance and the velocity profile for fully developed laminar and turbulent flows in smooth-walled tubes. A modification of the viscous damping that improves the predictions for non-Newtonian fluids is proposed. The presented $k-\epsilon$ predictions are in fairly good agreement with experimental data for the turbulent friction and the mean velocity profiles at various generalized Reynolds numbers and different values of the power-law index $n$.

A more conventional and general $k-\epsilon$ model was developed by Ro Kyoungchul and Ryou HongSun (2012) to analyze non-Newtonian fluid flows for more complex and various engineering problems. The modified $k-\epsilon$ model is based on the standard one with wall and damping functions including the drag reduction phenomenon. In order to validate their modified $k-\epsilon$ model, numerical simulations are performed for shear-thinning fluids, at different values of the flow index $0.4 \leq n \leq 1$. The predicted friction factors and mean axial velocity profiles agree well with the experimental results of literature (Dodge and Metzner (1959), Escudier and Presti (1998), Ptasinski et al. (2001)), and this agreement is much better than with the standard $k-\epsilon$ model. The proposed model also agree well with Malin's power law model in the simulation of blood flow. The authors indicate that the computational time and computer resources of the modified $k-\epsilon$ model are reduced by 
about one third of those required by the low Reynolds number $k-\epsilon$ models for power-law fluid (including Malin's model). The authors point out that their turbulence model better predicts the behavior of high power-law fluids.

Direct numeral simulation of turbulent pipe flows of shear-thinning fluids was carried out by Rudman et al. (2004) for $n=0.5,0.69$ and 0.75, using a spectral element-Fourier method, at a moderate Metzner-Reed Reynolds number $\left(R e_{M R} \simeq 3000\right.$ and 4000). $\mathrm{A}$ similar DNS study at a higher Meztner-Reed Reynolds number $\left(R e_{M R}=7500\right)$ was conducted by Rudman and Blackburn (2012). In the log-region, the velocity profile was shown to agree well with the experimental data by Rudman et al. (2001) and Rudman et al. (2002). The friction factors predicted by DNS were $10 \%$ to $15 \%$ higher than those referred to in earlier research (Dodge and Metzner correlations obtained from experiments). The authors reported that this is most likely related to the imperfect fit of the experimental data concerning fluids with power-law rheology. It was shown that, for a given Reynolds number, the flow deviates further from the Newtonian profile as the power-law index $n$ decreases, and the results suggest that the transition to turbulence is delayed. Moreover, the shear-thinning or thickening rheologies did not result in major changes to the nature of the flow at $R e_{M R}=7500$.

Direct numerical simulation (DNS) and large-eddy simulation (LES) are techniques well suited for predicting turbulent non-Newtonian fluid flows, because a detailed picture of the turbulent structures, profiles of turbulence energy, rms and Reynolds stresses are difficult to obtain experimentally. In DNS, numerically accurate and complete resolution of all spatial and temporal flow scales is required and no turbulence model is used. In LES, an accurate numerical resolution of a wide range of scales is required and only the smallest scales are modeled using a subgrid scale (sgs) turbulence model. While DNS is clearly a very useful tool for accurately simulating the turbulent flows, LES however can yield quantitatively accurate predictions at a computational cost which is significantly lower than the corresponding DNS one, since the effect of the smallest scales in LES is modeled and the mesh is relatively coarse. Moreover, when the Reynolds number is significant, LES provides an effective tool for predicting the effect of the flow index and Reynolds number on the turbulent fields of non-Newtonian fluids. 
There are very few studies employing LES for non-Newtonian fluids. To predict the turbulence features in non-Newtonian fluid flows, Ohta and Miyashita (2014) developed a turbulence model that can reproduce the DNS results. They pointed out that constructing a new turbulence model extended for non-Newtonian fluids would obviously be difficult, since the new model would have to consider additional terms in the filtered Navier-Stokes equations (i.e. it can hardly be expected to construct a turbulence model by introducing turbulence corrections to the additional terms). Therefore, they considered a different approach: they proposed an extended Smagorinsky model with a correction for the filter width of the locally varying viscosity. Ohta and Miyashita (2014) performed DNS and LES of turbulent channel flow, in two non-Newtonian fluids with the viscosity described by both the power-law model $(n=0.85$ and 1.15) and Casson's model. By performing LES with the Smagorinsky model as sgs model, extended according to the results of the DNS, they evaluated the reliability of the extended sgs model. They found that it could more accurately predict the velocity of turbulent flows of fluids described by both Casson's model and power-law model as compared to the standard Smagorinsky model (i.e. the results of LES with the extended model agree more with those obtained by DNS with high resolution). Consequently their study showed that the Smagorinsky model of non-Newtonian turbulent flows could be universally treated via a spatial scaling of the locally varying viscosity.

Thais et al. (2010) proposed an LES approach for viscoelastic turbulent channel flows, based upon a temporal deconvolution method (which was developed for LES of Newtonian channel flows) for residual Newtonian stress modelling and secondary regularization for unresolved subfilter Newtonian stress. A particular emphasis is put on the turbulent drag reduction. Excellent agreement is obtained between temporal LES and DNS in terms of drag reduction prediction. The first normal Reynolds stress component is exactly in line (the agreement is even better than observed for Newtonian flows), while shear is slightly overestimated in the near-wall region. The subfilter Reynolds stresses are marginally smaller than observed for a Newtonian flow at a moderate level of the drag reduction, while they are markedly smaller than observed for a Newtonian flow at a high level of the drag reduction.

Molla et al. (2012) carried out LES of a pulsatile transition-to-turbulent non-Newtonian blood flow of arterial stenosis, within a 3D-channel, using a new consistent modelling approach for the sgs stress. Their approach used the Cross model for the modelling of the 
molecular viscosity and the advanced dynamic non-linear sgs stress model (DNM) of Wang and Bergstrom (2005) for the modelling of the sgs stress tensor so that both the molecular and sgs shear stresses could be represented as non-linear functions of the strain rate tensor. In their research, a high Womersley number $(\alpha=10.5)$ and a low Reynolds number (Re $=1200$ ), both characteristics of blood flows in large arteries of humans and animals, were tested. The first and second order flow statistics of the velocity field were analysed. The streamwise velocity at different downstream locations agreed with the experimental results of Ahmed and Giddens (1983). The distributions of the other statistics (wall shear stress, RMS of velocity fluctuations, Reynolds and sgs stresses) were plotted but they were not compared with the results from the literature. The authors concluded that although their preliminary results were encouraging, further future studies were necessary to prove that their new modelling approach is an effective numerical tool for LES of non-Newtonian fluid flows.

The above literature survey indicates that the few DNS and LES devoted to turbulent pipe flows of power law fluids have been performed for $0.4 \leq n \leq 1.4$ at $R e_{M R}<7500$. The current study deals with LES of fully developed turbulent pipe flows of power-law fluids in a cylindrical pipe. The flow index $n$ ranges from 0.5 to 1.4, and the Reynolds number is up to 12000 . The aim of the current research is to investigate the effects of the power law index, $n$, and Reynolds numbers on turbulent power law pipe flows. The LES at the highest Reynolds number $\left(R e_{s}=12000\right)$ for different flow index values are new as well as the computation of several statistics such as :

- higher-order statistics (skewness, flatness),

- cross-correlation coefficients and kinetic energy,

- correlations for the peak position of the RMS of the velocity fluctuations (with respect to $n$ ) and of the kinetic energy.

This study is also intended to examine the effectiveness of the LES approach for predicting the turbulent pipe flow of power-law fluids.

The paper is organized as follows: section 2 provides the governing equations and the numerical procedure. The influence of the power law index and Reynolds numbers on many turbulent statistics (mean velocity profiles, root mean square of fluctuating velocities, Reynolds shear stress, high order statistics, friction factor) are discussed in section 3. 
The present LES predictions are also compared to the available results of literature for validation. The main conclusions are found in section 4 .

\section{Governing equations and numerical procedure}

\subsection{Governing equations}

The present study deals with the fully developed turbulent pipe flows of power-law fluids in a cylindrical pipe. The dimensionless filtered governing equations for incompressible nonNewtonian fluids, using the centerline axial velocity $\left(U_{c L}\right)$ of the analytical fully developed laminar profile and the pipe radius $R$ as velocity and length scales respectively, can be expressed as follows:

$$
\begin{gathered}
\frac{\partial \bar{u}_{i}}{\partial x_{i}}=0 \\
\frac{\partial \bar{u}_{i}}{\partial t}+\frac{\partial \bar{u}_{i} \bar{u}_{j}}{\partial x_{j}}=-\frac{\partial \bar{p}}{\partial x_{i}}+\frac{1}{R e_{s}} \frac{\partial}{\partial x_{j}}\left[\dot{\gamma}_{d}^{n-1}\left(\frac{\partial \bar{u}_{i}}{\partial x_{j}}+\frac{\partial \overline{u_{j}}}{\partial x_{i}}\right)\right]-\frac{\partial \overline{\tau_{i j}}}{\partial x_{j}}
\end{gathered}
$$

The Reynolds number of the simulations is defined as $R e_{s}=\frac{\rho U_{c L}^{2-n} R^{n}}{K}$. The apparent viscosity $\eta$ of the fluid is modelled by a power-law (Ostwald de Waele model) given by $\eta=K \dot{\gamma}^{n-1}$, where $K$ is the consistency, $n$ is the power-law index, and $\dot{\gamma}$ is the shear rate. The flow index values $0<n<1, n=1$ and $n>1$ correspond to shear thinning (or pseudoplastic), Newtonian, and shear thickening (or dilatant) cases respectively. The dimensionless shear rate is defined as: $\dot{\gamma}_{d}=\frac{\dot{\gamma} R}{U_{c L}}$. The above dimensionless equations are written in cylindrical coordinates and in terms of the variables $q_{r}=r v_{r}, q_{\theta}=r v_{\theta}$ and $q_{z}=v_{z}$ in the code to avoid the singularity at the axis pipe $(r=0)$. A mean pressure gradient, at each time substep, is evaluated to balance the friction losses (the mean pressure gradient in the $q_{z}$ equation maintains a constant bulk velocity). 


\subsection{Dynamic eddy viscosity model}

The subgrid stress tensor $\overline{\tau_{i j}}$ is related to the of strain rate tensor $\overline{S_{i j}}$ as

$$
\bar{\tau}_{i j}=-2 \nu_{t} \overline{S_{i j}}=-2 C_{d} \Delta^{2}|\bar{S}| \overline{S_{i j}}=-2 C_{d} \Delta^{2}\left[2 \overline{S_{i j}} \overline{S_{i j}}\right]^{1 / 2} \overline{S_{i j}}
$$

where the turbulent viscosity $\nu_{t}$ is expressed using an eddy viscosity assumption, $\Delta$ is the computational filter, $C_{d}$ is the model constant, and $\overline{S_{i j}}=\frac{1}{2}\left[\frac{\partial \overline{u_{i}}}{\partial x_{j}}+\frac{\partial \bar{u}_{j}}{\partial x_{i}}\right]$.

Following the model proposed by Germano et al. (1991), with modifications and extensions provided by Lilly (1992), the coefficient of the dynamic model $C_{d}$ is dynamically determined as :

$$
C_{d}=-\frac{1}{2 \Delta^{2}} \frac{\left\langle L_{i j} M_{i j}\right\rangle}{\left\langle M_{i j} M_{i j}\right\rangle}
$$

where the tensors $L_{i j}$ and $M_{i j}$ are given as follows

$$
\begin{gathered}
L_{i j}=\widetilde{\overline{\bar{q}}_{i} \overline{\bar{q}}_{j}}-\widetilde{\widetilde{q}_{i}} \widetilde{\bar{q}}_{j}=-2 C_{d} \Delta^{2} M_{i j} \\
M_{i j}=\frac{\widetilde{\Delta^{2}}}{\Delta^{2}}|\widetilde{\bar{S}}| \widetilde{\bar{S}_{i j}}-\mid \widetilde{\bar{S} \mid \bar{S}_{i j}}
\end{gathered}
$$

Here a test filter $\tilde{\Delta}$ larger than the grid filter $\Delta$ which is defined as $\Delta=(r \Delta r \Delta \theta \Delta z)^{1 / 3}$ is introduced. The total volume-average box filter in cylindrical coordinates is used. The $\tilde{\Delta}$ filter width is twice in the axial and azimuthal directions in comparison to the grid filter $\Delta$. The angle brackets, \langle\rangle , denote spatial averaging procedure along the homogeneous directions of the flow to make the subgrid-scale coefficients well conditioned, and to reduce a large part of the spatial variations. Negative values of the turbulent viscosity are eliminated (i.e. set to zero).

Note that in the present LES study, the standard dynamic model of Germano et al. (1991) and Lilly (1992) is used to model the sgs shear stresses. The sgs stress tensor is thus given as $\tau_{i j}=-2 \nu_{t} \bar{S}_{i j}$, where the sgs viscosity $\nu_{t}$ is a linear function of the shear rate $|\bar{S}|$ (cf. Eq.(3)). However, when the spatial filter is applied to the Navier-Stokes equations, 
in non-Newtonian viscous fluid flows (with spatially varying viscosity characteristics), additional terms are derived (Ohta and Miyashita, 2014) because the stress tensor is a non linear function of the strain rate tensor. This means that in the current LES study with a standard dynamic model, the additional terms are ignored. Indeed, these terms are smaller than the sgs stress: Ohta and Miyashita (2014) plotted the profiles of the sgs stress and of the additional terms that are estimated by filtering the DNS results of Newtonian and power-law fluids. From these profiles, they demonstrated that the additional terms are much smaller than the sgs stress. Therefore, in their study, they concentrated on a modification of the sgs model for the viscosity characteristics of non-Newtonian fluid and ignored the additional terms. Moreover, the grid resolution of the present LES approaches that of the true DNS (LES is performed with high resolution). The coarse DNS and LES with sgs model, both performed at the same grid resolution $\left(65^{3}\right)$, are in satisfactory agreement (see subsection 3.2). It seems that the grid scale is finer than the dominant scales of the flow so that it suffices to yield the right behaviour of the dominant scales (i.e. the dynamic sgs contribution is reduced and the LES results approach those of DNS). This provides justification for why it is reasonable to ignore the additional non linear terms in the filtered Navier-Stokes equations.

\subsection{Numerical procedures}

The governing equations were discretized on a staggered grid using cylindrical coordinates. The numerical integration was performed by a finite difference scheme, second-order accurate in space and time. The time integration and the velocity-pressure coupling are performed by the fractional step method suggested by Rai and Moin (1991) and modified by Verzico and Orlandi (1996). In a first step, the momentum equations are solved by an incremental factorization method of ADI type to compute a non solenoidal approximation of the velocity field. This intermediate velocity field is not locally divergence-free but it is globally because periodic boundary conditions are applied in $\theta$ and $z$ directions and the wall at $r=R$ is impermeable. In this step, the time schemes are a third-order RungeKutta explicit scheme and a Crank-Nicolson implicit scheme for the convective and diffusive terms, respectively. In the second step, the non-solenoidal velocity field is projected onto a 
solenoidal velocity field in order to correct the pressure and velocity fields and to satisfy the continuity equation on each cell. This projection step requires to solve a Poisson equation for a scalar pressure increment $\phi$. This Poisson equation is solved by Fast Fourier Transforms in the two periodic directions. This implies that the grids are necessarily uniform in the streamwise and azimuthal directions. In equation (2), the shear rate $\dot{\gamma}_{d}$ is calculated explicitly.

The pipe length in the streamwise direction is equal to $L_{z}=20 R$. In the radial direction, the grid distribution is stretched (with an hyperbolic tangent function). A large computational time has been spent checking the grid and $L_{z}$ independence of the results, especially on the axial velocity profiles. Different grids have been tested for $n=0.75,1$ and 1.2 and predictions were compared to those of the literature. The grid $65 \times 65 \times 65$ was found to provide an accurate prediction of turbulence statistics (in agreement with the available data of the literature) and to give a good compromise between the required CPU-time and accuracy.

In wall bounded flows, the near-wall streaks in the buffer region play an important role in the regeneration of turbulent energy. The maximum production of turbulent kinetic energy occurs in the inner layer. Consequently a finer mesh is required in the near-wall region. It is well known that a wall-resolved LES needs a near-wall grid resolution of $y^{+} \simeq 1$ (which means that the first gridpoint is located within the viscous sublayer) to capture the dynamically dominant streak-like vortical structures in the viscous and buffer regions. For present LES, the mesh with 65 nodes in the radial direction has a grid resolution of $y_{1}^{+}<1$ for the first grid cell adjacent to the wall (see table 1 and the following discussion). That means that the flow simulation is able to capture accurately the major part of the eddies that contribute to the momentum transport.

The two-point correlations of the fluctuating streamwise velocity along the axial and azimuthal directions provide information about the flow structures and whether the pipe length is sufficient to capture the largest eddies in the flow. In a previous work (Redjem, Phd Thesis, 2008), we shew that the computational domain $L_{z}=15 R$ is large enough to simulate the largest structures for $n=1$ (Newtonian fluid flow) at $R e \simeq 5000$. The computational domain in the periodic directions was not only large enough to capture the largest eddies of the flow, but also sufficient for the scalar field (i.e. to simulate the largest thermal structures). In the present LES, $L_{z}=20 R$ was fixed in all simulations. When the 
length is increased to $L_{z}=20 R$, a convergence to the statistical steady state is reached for $n>0.69$. In these cases, the results are obtained in a reasonably long pipe, rather than spending a large computational time checking the independence of the two-point correlations on $L_{z}$. For $n \leq 0.69$, the pipe length is also considered large enough even though the statistics are less good.

Rudman et al. (2004) performed DNS runs on a domain lengths equal to $4 \pi D$ for various $n$. Because their simulation for $n=0.5$ was in transitional regime, they also performed a DNS for $n=0.5$ on a domain length equal to $8 \pi D$. However, they observed that the average flow results of both DNS are very similar and still transitional for both domain lengths. The main difference is in the axial turbulence intensity that is slightly higher with the longest domain. The friction factors of the two domains differ by less than $2 \%$. It clearly appears that the structures do not fill the domains (isolated structures persist when the domain length of the simulation is increased). The authors concluded that the results on the extended domain confirm that this flow is transitional.

Table 1 lists the simulation parameters and mean flow quantities, at $R e_{s}=4000$. The first gridpoint, $y_{1}^{+}$, is also given in Table 1, for each simulation. This table shows that due to the non-uniform grid, the first gridpoint, $y_{1}^{+}$, in all cases is located at $y^{+}<1$. The next gridpoints are also within the viscous sublayer: from a minimum of 21 gridpoints at $n=0.5$ to a maximum of 29 gridpoints at $n=1.4$. For $R e_{s}=8000$ and 12000 , the first gridpoint $y_{1}^{+}$ is also located at $y^{+}<1$ and there are many gridpoints within the viscous sublayer: from a minimum of 18 gridpoints to a maximum of 25 (see tables 2 and 3). Thus the simulation run for the smallest Reynolds number and flow index $\left(R e_{s}=4000\right.$ and $\left.n=0.5\right)$ can be considered as LES with high resolution; for the other cases, the LES are of moderate resolution except at $R e_{s}=12000$ for $n=1.2$ which is rather a low LES resolution. Indeed, Montreuil (2000) pointed out that three grid resolution's levels can be distinguished: LES with $\Delta z^{+} \simeq 35$ and $(r \Delta \theta)^{+}<10$ is referred to as LES with high resolution; LES with $\Delta z^{+} \simeq 70-80$ and $(r \Delta \theta)^{+} \simeq 15-20$ is referred to as LES with moderate resolution; LES with $\Delta z^{+} \simeq 155$ and $(r \Delta \theta)^{+} \simeq 35-40$ is referred to as LES with low resolution. When employing schemes second-order accurate in time and space, good results can be derived with $\Delta z^{+}<100$ and $(r \Delta \theta)^{+}<12$ (Zahrai (1995)). Moreover, Zang (1991) showed that an accurate LES can 
be performed using $\Delta z^{+}<80$ and $(r \Delta \theta)^{+}<40$, with a minimum of three points within the viscous sublayer. In the present LES, there are many gridpoints within the viscous sublayer (more than three points) confirming that accurate LES are performed in this study.

To avoid the possible singularity at zero shear rate $(\eta$ is infinite when $\dot{\gamma}=0$ and $n<1)$, a cut-off value $\dot{\gamma}=10^{-6}$ is added in the code. Below this value, the shear rate is supposed constant and set equal to the cut-off value. However this cut off is never invoked in the present LES runs because $\dot{\gamma}$ is always above the cut off value.

The time is made dimensionless using the pipe radius, $R$, and the maximum velocity of the laminar power law profile, $U_{c L}$. The calculations have been conducted at the constant Courant, Friedrichs and Lewy condition: $\mathrm{CFL}=1.7$. In this case, the time step is computed from the imposed CFL, which is however limited by the value $0.08 R / U_{c L}$ for shear-thickening and Newtonian fluids, and by $0.04 R / U_{c L}$ or $0.01 R / U_{c L}$ for shear-thinning fluids, in order to avoid large time discretization errors. The simulation is stopped if the computed time step size becomes very small (which means that the solution is diverging). This occurs when decreasing flow index $n$ : it was necessary to reduce the time step DT to avoid very large (diverging) velocity fields from the dimensionless time step DT $=0.08$ (for $n>0.75$ ) to $\mathrm{DT}=0.04$ (for $n=0.75$ ) and $\mathrm{DT}=0.01$ (for $n<0.75$ ). Hence, in the present LES, the time step varies from $0.01 R / U_{c L}$ to $0.08 R / U_{c L}$.

The statistics are computed by averaging in the periodic directions and in time. The final data are obtained by ensemble averaging over the time interval 6750 (i.e. from the dimensionless $t=250$ until $t=7000)$ for the smallest flow index $(n=0.5$ and 0.69$)$. At higher flow index $(n>0.69)$, the integrated time over which the statistics are collected is 9750 (i.e. from the dimensionless time $t=250$ until $t=10000$ ).

\section{Results and discussion}




\subsection{Reynolds numbers}

First of all, note that the Metzner-Reed Reynolds number, $R e_{M R}$, is computed and not imposed in each simulation (the imposed Reynolds number is $R e_{s}$ ). The relation between the two Reynolds numbers is:

$$
\frac{R e_{s}}{R e_{M R}}=\frac{1}{8}\left(3+\frac{1}{n}\right)^{n}\left(\frac{U_{c L}}{U_{b}}\right)^{2-n}
$$

If the flow is laminar, Eq. (7) reads:

$$
\frac{R e_{s}}{R e_{M R}}=\frac{1}{8}\left(\frac{3 n+1}{n+1}\right)^{2}\left(\frac{n+1}{n}\right)^{n}
$$

As a consequence, for laminar flows, the ratio $R e_{s} / R e_{M R}$ monotonically increases from $R e_{s} / R e_{M R}=0,601$ for $n=0.5$ (the most shear thinning fluid flow in this study) to $R e_{s} / R e_{M R}=1,248$ for $n=1.4$ (the most shear thickening fluid flow), passing through $R e_{s} / R e_{M R}=1$ for $n=1$ (Newtonian fluid flow). In other words, at fixed $R e_{s}$, the values of $R e_{M R}$ decrease when $n$ increases.

On the other hand, from Tables 1-3, one can note that for turbulent flows at fixed $R e_{s}$, the values of $R e_{M R}$ decrease when $n$ varies from 0.5 to 1 , but increase when $n$ increases from 1 to 1.4. This is due to the variation of the dimensionless bulk velocity $\left(U_{b} / U_{c L}\right)$ with $n$ in equation (7) : both $U_{b} / U_{c L}$ and the centerline velocity $\left(U_{c} / U_{c L}\right)$ vary in a similar way to $\operatorname{Re}_{M R}$ in Tables 1-3.

Mishra and Tripathi (1971) derived a general criterion to characterize the transition between laminar and turbulent flows in non-Newtonian fluid flows. This criterion is based on the critical Reynolds number :

$$
R e_{c r}=2100 \frac{(4 n+2)(5 n+3)}{3(3 n+1)^{2}}
$$

Using this criterion, one can calculates $R e_{c r}$ for each $n$. Table 1 shows that all the simulations are performed for turbulent flows. 


\subsection{Mean velocity profile}

The laminar axial velocity profiles, for various flow indexes $n$ at $R e_{s}=500$, are depicted in figure 1, along with the analytical distribution:

$$
\frac{U_{z}}{U_{b}}=\frac{3 n+1}{n+1}\left[1-\left(\frac{r}{R}\right)^{\frac{n+1}{n}}\right]
$$

The predictions demonstrate a good agreement with the analytical equation. When $n<1$, the velocity profiles flatten at the pipe center because the apparent viscosity $\eta$ increases where the strain rate $\dot{\gamma}$ decreases, and the fluid tends to behave as a solid. Opposite behaviours are observed for $n>1$ or close to the wall where $\dot{\gamma}$ increases. Indeed, for dilatant fluids $(n>1)$, the apparent viscosity increases with increasing shear rate, i.e. near the wall. A possible explanation of the dilatant behavior is as follows. This behaviour is encountered in concentrated suspensions of solid particles. At rest, the voidage is at a minimum and the liquid present is only sufficient to fill the void space. At low shear rates, the liquid lubricates the motion of each particle past the others thereby minimizing solid-solid friction. Consequently, the resulting stresses are small. At high shear rates, however, the mixture slightly expands (dilates) so that there is no longer sufficient liquid to fill the increased void space and prevent direct solid-solid contacts and friction. This leads to the development of much large shear stresses (which result in increased friction) than those seen in a pre-dilated sample at low shear rates. This mechanism causes the apparent viscosity to rise rapidly with increasing rate of shear (Rajendra P. Chhabra, 2010).

Shear thinning fluids $(n<1)$ are generally high polymer solutions or many suspensions. With increasing shear rates, the molecules are progressively aligned and these fluids become less viscous with increasing shear rates. The micro-structure of such materials is even smashed up at higher shear. This results in lower viscosities, hence the fluid flows more easily. Naturally, smaller the value of $n$ is, more shear thinning the material is.

Validation of the present LES calculations of the velocity field is also achieved by comparing the present results to the following results of literature for fully developed turbulent pipe flows:

- the Laser Doppler Anemometry (LDA) and Particle Image Velocimetry (PIV) measurements by Eggels et al. (1994), at $R e_{b}=U_{b} D / \nu=5300$ for $n=1$, 
- experimental data by Pinho et al. (1990) for a concentration of a polymer CMC (sodium CarboxyMethyl Cellulose) with $n=0.9$ at $R e_{w}=5070$,

- DNS predictions by Eggels et al. (1994) for $n=1$ at $R e_{b}=5300$,

- DNS results by Rudman et al. (2004) for $n=0.75$ at $R e_{M R}=3935$.

We first present the comparisons with the turbulent profiles measured by Eggels et al. (1994) for $n=1$ at $R e_{b}=5300$ and by Pinho et al. (1990) for $n=0.9$ at $R e_{w}=5070$ (see Fig.2a). Plotted on these graphs are the mean axial velocity distributions scaled by the friction velocity $\left(U^{+}=U / U_{\tau}\right.$ with $\left.U_{\tau}=\left(\tau_{w} / \rho\right)^{1 / 2}\right)$ as a function of the distance from the wall (based on the friction velocity and the mean wall viscosity, $y^{+}=\rho U_{\tau}(r-R) / \eta_{w}$ ). The relation between the pressure gradient and the wall shear stress gives the friction velocity $U_{\tau}$ necessary to scale the axial velocity in wall units. The mean wall viscosity $\eta_{w}\left(\eta_{w}=K \gamma_{w}^{n-1}\right)$ necessary to scale the distance from the wall $\left(y^{+}\right)$is obtained from the simulation results. The axial velocity distribution is slightly underestimated in the log-region in comparison with this experimental data. This discrepancy may be due to the difference in the Reynolds number values between the present LES and the experiments of literature. Indeed, $R e_{s}=$ 4000 corresponds to $R e_{b}=4449$ for $n=1$ (see Table 1 ), and it corresponds to $R e_{w}=5200$ for $n=0.9$.

On the other hand, the predicted LES profile for a flow index $n=0.75$ at $R e_{s}=4000$ indicates a good agreement with the DNS result by Rudman et al. (2004)(see Fig. 2b). The influence of the power-law index $n$ on the velocity profile is also illustrated on this graph. In the viscous sublayer $\left(0 \leq y^{+} \leq 5\right)$, the normalized velocity profile agrees with the universal linear law $U^{+}=y^{+}$, for all values of $n$ except for $n=0.5$ where the prediction is slightly overestimated. This means that this flow is in the transitional regime and it confirms the observation by Rudman et al. (2004) for $n=0.5$. Indeed, for $n=0.5$, the viscosity is higher in the core region and hence the turbulence is not as fully developed here, even though the Reynolds number $\left(R_{M R}=5302\right)$ is slightly higher than that observed for the other flow index values (see Table 1). This may be due to the difference between the viscosity and Reynolds number evolutions: the viscosity increases more rapidly than the Reynolds number for more shear-thinning fluid flows, damping the turbulence and inducing a less well-developed turbulent flow. In the log-region $\left(y^{+}>30\right)$, the mean axial velocity profile for $n=1$ agrees with the $\log$-law $U^{+}=2.5 \ln \left(y^{+}\right)+5.5$, and departs from the Newtonian 
behaviour when $n$ is different from unity. This deviation is more pronounced for $n<1$ as the flow index $n$ decreases. Similar trends have been observed by Rudman et al. (2004).

The influence of the Reynolds number is shown in Fig. 2c. By fixing the power-law index $n$ and increasing $R e_{s}$ both an enhancement of the axial velocity values is clearly seen in the log-region, and the log-region is more expanded. Moreover, for a given Reynolds number, the log-region is more expanded by decreasing $n$.

Finally, note that the grid resolution and the sgs model play a non negligible role, Fig. 2d-e. This can be observed by comparing LES with and without the sgs model, at the highest Reynolds number $\left(R e_{s}=12000\right)$, for two flow indexes $(n=0.8$ and $n=1.2)$, using various meshes, from a very coarse grid $(17 \times 30 \times 9)$ to a finer grid $(65 \times 65 \times 65)$. For the coarser grid, the predictions for the axial velocities without the sgs model deviate from those with the sgs model, especially for $n=0.8$, indicating how important this model is. Indeed it enables an improvement in the results when very coarse grids are used, with the LES (with dynamic model) having significantly lower centerline velocity than the no sgs model (the sgs model provides more damping as expected). For the intermediate and finer grids, there is no difference between the LES results with and without sgs model. It seems that the sgs model is not important and that the fine grid resolution (65 $5^{3}$ ) is potentially good enough for a DNS. The role of the sgs model is reduced when the resolved viscous term (ie the gamma term) in Eq.(2) is greater than that due to the sgs one (ie the $\tau_{i j}$ term). If the near wall structures are more oredered (ie more viscous and less turbulent), the sgs model should be less important because less energy goes from the resolved to the unresolved scales. Note that the ratio between the centerline velocities using LES with dynamic model for the coarser and finer grids is approximatly 44/23 for $n=0.8$ and approximatly 33/18 for $n=1.2$. These ratios denote a large difference between the centerline velocities, especially for $n=0.8$, due to the large difference between the corresponding friction velocities: the friction velocity obtained with the coarser grid is almost twice that calculated with the finer grid. This may be due to the choice of the grids : LES with resolution less than $65^{3}$ should never be performed for any kind of circular pipes (one can never generates reasonable velocity oscillations in the azimuthal direction in a domain wide $2 \pi$ with 32 points). In the simulations with $65^{3}$, the grid scale is fine enough to be able to simulate the dominant scales of the flow and its right behaviour, as previously discussed. Similar conclusions have 
been reported for Newtonian fluid flows by Feiz et al. (2003).

\subsection{Friction factor}

The friction factor is defined by $f=2 \tau_{w} /\left(\rho U_{b}^{2}\right)$. For the fully developed laminar flows simulated at $R e_{s}=500$ for various $n$ values, the predicted friction factor value is $f \simeq$ 0.0341. This is within a $2.3 \%$ accuracy when compared with the well-known correlation $f=16 / R e_{M R}$. On the other hand, for the fully developed turbulent flows, the friction factor decreases for decreasing $n$ (at a given $R e_{s}$ ) and for increasing $R e_{s}$ (at a given $n$ ), see Table 1. The present LES predictions of $f$ for shear-thinning and Newtonian fluids $(n \leq 1)$, at $R e_{s}=4000$, are slightly overestimated in comparison to the following Dodge and Metzner (1959) correlation:

$$
f_{D M}=\frac{a}{R e_{M R}^{b}}
$$

where $a=0.0665+0.01175 n$ and $b=0.365-0.177 n+0.062 n^{2}$. The discrepancies vary between $9 \%$ and $14.5 \%$ for the cases at $R e_{s}=4000$ (see Table 1), between $2.2 \%$ and $20.6 \%$ at $R e_{s}=8000$ (see Table 2), and between $4.4 \%$ and $15.8 \%$ at $R e_{s}=12000$ (see Table 3). A similar observation has been reported by Rudman et al. (2004): their DNS predictions were $10 \%$ to $15 \%$ higher than the Dodge and Metzner correlation. For shear-thickening fluids, the discrepancy is enhanced. Gomes (1987) proposed an explicit relation for pseudo-plastic fluids $(n \leq 1)$ :

$$
f_{G}=0.110 n^{0.616} R e^{-0.287}
$$

Table 1 compares the LES predicted friction factors to the above mentioned correlation. This relationship seems to give better results for $R e_{s}=4000$ : the present friction factor is now $3.7 \%$ to $10.4 \%$ higher than Gomes correlation for $n \leq 1$. There are a number of other friction factor correlations (empirical or semi-empirical) for pipe flows of power law fluids, in the literature. El-Emam et al. (2003) tested correlations against published data. They noted that many of these correlations fitted the experimental data of their authors but did not fit that of other authors, indicating a lack of generality. 


\subsection{Mean normalized viscosity}

The variation of the mean viscosity scaled by the mean wall viscosity, versus the shear rate, for different values of power law index $n$, at $R e_{s}=4000$, can give further insights into the flow field. The smallest values of the relative shear rate $\langle\dot{\gamma}\rangle / \dot{\gamma}_{w}$ in figure 3a are located in the pipe centre, while the highest ones are located near the pipe wall. For the flow index $n=1$, the Newtonian behaviour is find (constant viscosity equal to wall viscosity). At $n<1$, this graph (Fig. 3a) clearly displays lower relative viscosity $\langle\eta\rangle / \eta_{w}$ for high shear rates. For a given $n(n<1)$, the relative viscosity increases from the pipe wall towards the pipe centre because the fluid tends to behave like a solid rather than a liquid when approaching the core region of the pipe, due to the lower shear rate in this region. This trend is more pronounced as $n$ decreases. For $n>1$, the opposite behaviour is observed. Shear thickening fluids become more viscous with increasing shear rate. Shear thickening behaviour is less common and generally arises in fluids that have a highly regular micro-structure at rest. When the fluid begins to move, the micro-structural components jam against each other, thickening the fluid thus preventing movement.

In the core region, for flows with increasing shear-thinning behavior $(n<1)$, the deviation from the Newtonian behaviour is faster than that for flows with increasing shear thickening behaviour. The ratio between the viscosity in the centre and the mean wall viscosity is about 1.8, 2.2, 2.8 and 5.1 for the flow index $n=0.8,0.75,0.69$, and 0.5 respectively, and approximately 0.6 and 0.4 for $n=1.2$ and 1.4 respectively, indicating higher relative viscosities $\left(\eta / \eta_{w}\right)$ in the core region of the pipe for $n<1$ than for $n>1$. Note that for all values of $n$, the mean viscosity remains constant in the viscous sublayer up to $y^{+} \simeq 5$, denoting a linear velocity distribution in this zone (not shown here).

Figure $3 \mathrm{~b}$ depicts the behaviour of the dimensionless apparent viscosity $\langle\eta>$ versus the wall distance $y^{+}$, for $R e_{s}=4000$ : near the wall, the apparent viscosity $\langle\eta\rangle$ is constant for a given $n$ up to $y^{+} \simeq 5$. It increases with increasing $n$ for $y^{+}<5$, while it decreases with increasing $n$ after the wall distance $y^{+} \simeq 35$, for $n \geq 0.69$. Once again, the case $n=0.5$ deviates from this behaviour probably because this flow is transitional. 
When plotting the mean relative viscosity $\langle\eta\rangle / \eta_{w}$ versus the mean relative shear rate $<\dot{\gamma}>/ \dot{\gamma}_{w}$, Fig.3c, all the distributions collapse, as expected. Indeed, the dimensionless apparent viscosity, $\eta_{d}$, is related to the dimensionless shear rate and the Reynolds number by $<\eta_{d}>=<\dot{\gamma}_{d}^{n-1}>/ R e_{s}$. Therefore, the Reynolds number disappears in the relative viscosity $<\eta_{d}>/ \eta_{d, w}=<\eta>/ \eta_{w}=<\dot{\gamma}^{n-1}>/ \dot{\gamma}_{w}^{n-1}$ However, the plot of the mean relative viscosity $\langle\eta\rangle / \eta_{w}$ versus the mean shear rate $\langle\dot{\gamma}\rangle$ clearly exhibits the effect of the Reynolds number on $\langle\eta\rangle / \eta_{w}$, Fig.3d : the mean relative viscosity $\langle\eta\rangle / \eta_{w}$ is noticeably affected by the Reynolds number for $n=0.8$; the influence of $\operatorname{Re}_{s}$ (and thus $\left.R e_{M R}\right)$ on the viscosity is quite small for $n=1.2$; with increasing $R e_{s}$, the mean viscosity becomes almost independent of $R e_{s}$ (the LES predictions at $R e_{s}=8000$ and $R e_{s}=12000$ are very close to each other).

\subsection{Root mean squares of turbulent fluctuations and Reynolds stress}

In figure $4 \mathrm{a}$, the rms of axial, radial and azimuthal velocity fluctuations and the corresponding experimental data of Eggels et al. (1994) are in reasonable agreement for $n=1$. In figure $4 \mathrm{~b}$, for $n=0.9$, the LES predictions are underestimated in comparison to the experimental data by Pinho et al. (1990), particularly for the axial component near the wall.

The root mean square ( $\mathrm{rms}$ ) of fluctuating axial velocity is plotted in figure $5 \mathrm{a}$, at various values of power law index $n$, for $R e_{s}=4000$. The predicted $\mathrm{rms}$ for $n=0.75$ is in satisfactory agreement with the DNS result of Rudman et al. (2004). The maximum value of the axial velocity fluctuations increases and its position moves far away from the wall with increasing $n$. It moves from $y^{+} \simeq 13,67$ at $n=0.5$ to $y^{+} \simeq 23,79$ at $n=1.4$. The rms of axial velocity fluctuations, Fig. $5 \mathrm{a}$, is reduced in the vicinity of the wall when $n$ increases, and is enhanced after reaching the peak location. This is due to the fact that the apparent viscosity $\eta$ increases with increasing $n$ near the wall (in the viscous sublayer), but it decreases when $n$ increases in the turbulent layer (see Fig. 3c). Thus the turbulent fluctuations develop and are more intense far from the wall (after the peak) when $n>1$ 
and closer to the wall when $n<1$.

The radial and azimuthal velocity fluctuations are smaller than the axial ones, and reach a maximum further away from the wall, Fig. 5b-c. Both radial and azimuthal turbulence intensities diminish with decreasing $n$ in the turbulent layer (as soon as $y^{+}>20$ ), denoting an augmentation of the mean apparent viscosity in this region. For $n<1$, the increase of the mean viscosity towards the pipe core generates larger and weaker axial vortices, hence reducing the wall drag. For shear-thickening fluid flows, the mean viscosity is smaller in the pipe core, leading to less dissipation and shorter turbulent structures in this zone.

Figure 6a shows that for a given flow index $n$, when the Reynolds number increases, the distribution of the fluctuating axial velocity rms shifts towards the wall, and a significant rise of its peak is observed. The influence of $n$ on the peak value of axial velocity fluctuations appears to be more pronounced at the lowest Reynolds number (here $R e_{s}=4000$ ). At $R e_{s}=12000$, the flow index $n$ seems to have no effect on the maximum value of axial turbulence intensity, while its influence on the peak location is marked.

Similarly, on figures 6b-c, the distribution of the rms of radial and azimuthal velocity fluctuations moves towards the wall with increasing Reynolds number, since the thickness of the viscous sublayer diminishes and hence the turbulence transports higher momentum towards the wall. However, the maximum value of the radial velocity fluctuations is reduced when $R e_{s}$ increases, whereas the peak of the azimuthal turbulence intensity profile disappears, Fig. 6c, creating two new peaks at $R e_{s}=12000$.

The predicted Reynolds shear stress $\left\langle u_{r}^{\prime} u_{z}^{\prime}>/ U_{\tau}\right.$ agree reasonably with the experimental data $(n=1)$ and DNS results $(n=0.75)$ of literature, Fig. 7a. The Reynolds shear stress behaves like the axial turbulence intensity when the flow index varies (see Fig. 5a). Its distribution is shifted towards the wall with increasing $n$ for a given $R e_{s}$, and its peak value is noticeably enhanced. Similar behaviour is observed when the Reynolds number increases, for a given $n$, Fig. 7b. The influence of $R e_{s}$ on the Reynolds shear stress is much more pronounced for $y^{+}<40$ (i.e. before reaching the peak location) than for $y^{+}>40$. 
Figure 8 depicts the distribution of the cross-correlation coefficient of the Reynolds shear stress, $R_{u_{r}^{\prime} u_{z}^{\prime}}$, with respect to $y^{+}$. This graph exhibits a plateau with almost the same value for all $n$. The plateau is more and more extended with decreasing flow index $n$. This result means that the axial velocity fluctuations are correlated to the radial ones along a more extended region when $n$ decreases.

The effect of the power law index $n$ on the turbulence kinetic energy was also analyzed but is not shown here. The position of the maximum turbulent kinetic energy moves far further from the wall as $n$ increases, at a given Reynolds number. This behaviour is expected since the mean viscosity decreases towards the pipe centre with increasing $n$. When $R e_{s}$ increases, the peak value of kinetic energy is enhanced.

Figure 9 depicts the peak position $\left(y^{+}\right)$of the rms of velocity components, as well as the peak position of the turbulent kinetic energy, versus the flow index $n$, at $R e_{s}=4000$. All the peak positions shift towards the core region with increasing $n$. The graph confirms that the maximum of the axial velocity component is always closer to the wall pipe than those of the wall-normal and azimuthal velocity components, irrespective of the flow index $n$. The predicted values of the peak position of $<u_{r}^{\prime 2}>^{1 / 2}$ and that of $\left\langle u_{\theta}^{\prime 2}>^{1 / 2}\right.$ can be interpolated by $y^{+}=82.8 n^{0.29}$ and $y^{+}=67.2 n^{0.47}$ respectively. The peak position of $<u_{z}^{\prime 2}>^{1 / 2}$ can be interpolated by the correlation $y^{+}=19.0 n^{0.72}$. Nearly the same $n$ dependency is observed for the peak position of kinetic energy: $y^{+}=20.0 n^{0.69}$, indicating the large contribution of the axial component on the turbulent kinetic energy. Keeping in mind the viscous sublayer thickness $\left(y^{+} \simeq 5\right)$ and that of the logarithmic region $\left(y^{+} \geq 30\right)$, the present predictions show that for $0.5<n \leq 1.4$, the maximum of the rms of axial velocity as well as the maximum of kinetic energy are reached in the buffer layer. The peak positions of $\left\langle u_{r}^{\prime 2}>^{1 / 2}\right.$ and $<u_{\theta}^{\prime 2}>^{1 / 2}$ are mainly located in the log-region, for all values of $n$. 


\subsection{Higher-order statistics}

A description of a turbulent variable at a given location and time is given by the probability density function (PDF) or the moments of the variable derived from the PDF. The skewness is the third moment of the fluctuation $v^{\prime}=v-\langle v\rangle$ normalized by the variance. A PDF which is symmetric (a Gaussian PDF) about the mean $\langle v\rangle$ will have zero skewness. All higher odd moments of such a symmetric PDF will also be identically zero. The skewness reveals information about the asymmetry of the PDF. A non-zero skewness indicates a skewed or asymmetric PDF, which in turn means that larger excursions in one direction are more probable than in the other. Positive skewness indicates that the PDF has a longer tail for $(v-<v>)>0$ than for $(v-<v>)<0$. Hence a positive skewness means that variable $v^{\prime}$ is more likely to take on large positive values than large negative values. A time series with long stretches of small negative values and a few instances of large positive values, with zero time mean, has positive skewness.

The kurtosis (or flatness) is the fourth moment of $v^{\prime}$ normalized by the variance. the kurtosis can be used as an indication of the tails of a PDF. A higher kurtosis indicates that relatively larger excursions from the mean are more probable. A PDF with longer tails will have a larger kurtosis than a PDF with more narrow tails. A time series with most measurements clustered around the mean has low kurtosis; a time series dominated by intermittent extreme events has high kurtosis. For the Gaussian behavior, the flatness value is equal to $F=3$.

The profiles of the skewness coefficients $S\left(u_{i}^{\prime}\right)=\frac{\left\langle u_{i}^{\prime 3}\right\rangle}{\left\langle u_{i}^{\prime 2}\right\rangle^{\frac{3}{2}}}$, at $R e_{s}=4000$, have been computed and are presented on Fig. 10a-b.. As the power law index $n$ decreases, the skewness coefficient of the three velocity components grows rapidly at the wall, indicating a more important intermittent (non Gaussian) behaviour with decreasing $n$, especially for the radial velocity fluctuations (not shown here). This indicates that large positive values of the velocity components rather than large negative values predominate near the wall. Far from the wall, irrespective of the flow index $n$, the skewness of $u_{r}^{\prime}$ quickly tends to the Gaussian value $\left(S\left(u_{r}^{\prime}\right)=0\right)$ towards the pipe centre, meaning that positive values of the variable are as probable than negative values. On the contrary, the skewnesses $S\left(u_{z}^{\prime}\right)$, 
Fig. 10a, and $S\left(u_{\theta}^{\prime}\right)$ (not shown here) go to zero towards the core region more rapidly with increasing $n$. They tend to zero further from the wall with an augmentation of $R e_{s}$, for each given $n$, Fig. $10 \mathrm{~b}$.

Profiles of the flatness coefficients $F\left(u_{i}^{\prime}\right)=\frac{\left\langle u_{i}^{\prime 4}\right\rangle}{\left\langle u_{i}^{\prime 2}\right\rangle^{2}}$ are sketched in figures 11a-c, at $R e_{s}=4000$. Close to the wall, the flatness factors $F\left(u_{z}^{\prime}\right)$ and $F\left(u_{r}^{\prime}\right)$ are significantly dependent on the flow index $n$. The rapid increase in the flatness factor near the wall reflects the high intermittency of the velocity fluctuations close to the wall. Such high values are attributed to the strong sweep events near by the wall (Xu et al., 1996). The intermittent behavior is more important as $n$ decreases, and more pronounced for $F\left(u_{r}^{\prime}\right)$ than for $F\left(u_{z}^{\prime}\right)$ and $F\left(u_{\theta}^{\prime}\right)$, Fig 11a-c. The more intermittent behaviour close to the wall means that the probability of observing large variations from the mean axial and radial velocity components close to the wall is much higher than in the centre of the pipe, especially when $n$ decreases. Far from the wall, the factor $F\left(u_{r}^{\prime}\right)$ is equal to the Gaussian value $(F=3)$, irrespective of $n$, whereas $F\left(u_{z}^{\prime}\right)$ and $F\left(u_{\theta}^{\prime}\right)$ tend to the Gaussian behaviour for only $n \geq 1$. The flatness profiles extends towards the core region with decreasing $R e_{s}$, for a given $n$, Fig. 12 .

\subsection{Flow pictures}

To explore the effects of the flow index on the near-wall structures, contours of the axial velocity at the distance $y^{+} \simeq 15$ from the wall are visualized in figures $13 \mathrm{a}$-d for $R e_{s}=4000$. As $n$ increases, the turbulence structures are more random, the axial correlation distance (stretching) is shorter and the streaks are shorter and stronger. This is in accordance with increasing turbulence intensities, from Fig.13a to Fig. 13d, meaning that the turbulence is more developed. For the lowest flow index $(n=0.5)$, Fig.13a, the turbulence activity is poor. This flow seems not fully developed, because of the higher viscosity in the core region. However, the mean flow statistics (of first and second order) are still accurately predicted. Contours of the resolved axial velocity in $r-\theta$ plane at $R e_{s}=4000$, Fig. 14 a-d, show that

the turbulent structures are essentially located close to the pipe wall for $n>0.5$. Further from the wall (for $y^{+}>15$ ), the turbulence activity is attenuated in comparison to that at 
$y^{+} \simeq 15$, especially for $n<1$. Indeed, in the core region, the viscosity is increased for $n<1$ (since this zone is a lower shear region) inducing a damping of the turbulent structures and hence a reduction in radial momentum transfer: the turbulent structures are weaker and bring less high speed fluid from the core region to the wall region. At $n=0.5$, when the Reynolds number is higher $\left(R e_{s}=8000\right.$, not shown here), the wall streaks are less long than those observed at the same position for $R e_{s}=4000$, denoting a more developed turbulence.

\section{Conclusion}

Detailed characteristics of fully developed turbulent pipe flows of power law fluids have been numerically explored, using large eddy simulation with dynamic subgrid-scale model, to investigate the power law index and Reynolds number effects on the velocity field. The Reynolds numbers $\left(R e_{s}=4000,8000\right.$ and 12000) and power law index $(0.5 \leq n \leq 1.4)$ have been considered. The main new results (in comparison to those in the literature) obtained in this study are:

- LES at a higher Reynolds number $\left(R e_{s}=12000\right)$ for different flow indexes $n$, - analysis of higher-order statistics,

- analysis of cross-correlation coefficients and kinetic energy, - establishment of correlations for the peak position of the RMS of the velocity fluctuations (with respect to $n$ ) and for the kinetic energy.

The present LES predictions and the available literature results agree quite well. In the fully developed turbulent flow, the log-region of the mean velocity profile is more expanded with decreasing flow index and increasing Reynolds number. The normalized velocity profile agree with the universal linear law, in the viscous sublayer, for all values of $n$ except for $n=0.5$. In this case, the flow is transitional; the delay in the transition to turbulence is caused by the shear-thinning rheology and a high viscosity in the flow core. More generally, the results allow to quantify the suppression of turbulence fluctuations with decreasing $n$ in the core region, after the peak position, of the RMS of velocity fluctuations. The peak position of all the rms shifts towards the core region with increasing $n$. Correlations to 
obtain these peak positions are proposed. At $R e_{s}=4000$, the maximum of the rms of axial velocity $\left\langle u_{z}^{\prime 2}>^{1 / 2}\right.$ as well as the maximum of kinetic energy are reached in the buffer layer, while the peak positions of $\left\langle u_{r}^{\prime 2}\right\rangle^{1 / 2}$ and $\left\langle u_{\theta}^{\prime 2}\right\rangle^{1 / 2}$ are mainly located in the log-region, for all values of $n$. The rapid increase in the flatness factor with decreasing $n$ near the wall reflects the high intermittency of the velocity fluctuations close to the wall. With increasing flow index and increasing Reynolds number, the axial stretching of the turbulent structures is reduced, leading to a better developed turbulence. A reduced friction factor for decreasing $n$ (at a given $R e_{s}$ ) and for increasing $R e_{s}$ (at a given $n$ ) is also shown. The apparent viscosity $\langle\eta\rangle$ is constant for a given $n$ up to $y^{+} \simeq 5$. It increases with increasing $n$ for $y^{+}<5$, while it decreases with increasing $n$ after the wall distance $y^{+} \simeq 35$, for $n \geq 0.69$.

The LES results obtained in this study indicate that LES, with the conventional dynamic model of Germano et al. (1991) and Lilly (1992) for evaluating the sgs stresses, using a fine grid, reproduced most the features of the non-Newtonian fluid flows, and thus seems capable of predicting non-Newtonian fluid flows and gaining a comprehensive understanding of the space and time dynamics of such flows, at a reduced computational cost (without resorting to any tuning of the sgs turbulence model). Furthermore, the present predictions could be useful in developing a low-Reynolds number turbulence model for predicting non-Newtonian fluid flows. Additional studies could be performed in the future to further explore whether a new modeling approach, in which the molecular and sgs stresses would be represented as non-linear functions of the strain rate tensor, will bring any additional improvement to LES of non-Newtonian fluid flows.

\section{Acknowledgments}

The authors would like to thank the reviewers for their useful comments. 


\section{REFERENCES}

Dodge, D.W. and Metzner, A.B., 1959. Turbulent flow of non-Newtonian systems. AIChE J. 5, 189-204.

El-Emam, N., Kamel, A.H., El-Shafei, M. and El-Batrawy, A., 2003. New equation calculates friction factor for turbulent flow of non-Newtonian fluids. Oil and Gas Journal 101, 36, 74-83.

Feiz, A.A., Ould-Rouiss, M. and Lauriat, G., 2003. Large eddy simulation of turbulent flow in a rotating pipe. Int. J. Heat Fluid Flow 24, 412-420.

Germano, M., Piomelli, U. and Cabot, W. H., 1991. A dynamic subgrid-scale eddy viscosity model. Phys. Fluids A 3, 1760-1765.

Gomes, F.J.A.D., 1987. Hydraulic, Power Law models, calculation method using rational polynomial models. Technical Meeting on Software Developing in Drilling Operations, CAPER/87, Salvador, Brazil.

Lilly, D. K., 1992. A proposed modification of the Germano subgrid-scale closure method. Phys. Fluids A 4, 633-635.

Malin M.R., 1997. Turbulent pipe flow of power-law fluids. Int. Comm. Heat Mass Transfer 24, $n^{\hat{A} \circ} 7,977-988$.

Mishra, P., Tripathi,G. 1971. Transition from laminar to turbulent flow of purely viscous non-Newtonian fluids in tubes. Chemical Engineering Science 26, 915-921.

Montreuil, E., 2000. Simulation numérique pour l'aérothermique avec des modèles sousmaille, $\mathrm{PhD}$ thesis, Université Pierre et Marie Curie, France.

Molla, M.M., and Paul, M.C., 2012. LES of non-Newtonian physiological blood flow in a model of arterial stenosis. Medical engineering and Physics 34 (8), 1079-1087.

Ohta, T., and Miyashita, M., 2014. DNS and LES with an extended Smagorinsky model for wall turbulence in non-Newtonian viscous fluids. J. Non-Newtonian Fluid Mech. 206, 29-39.

Rai, M.M., Moin, P., 1991. direct simulations of turbulent flow using finite-difference schemes. J. of Comp. Phys. 96, 15-53.

Rajendra P. Chhabra, 2010. Chapter 1, non-Newtonian Fluids : an introduction. Rheology of complex fluids. Abhijit P. Deshpande, J. Murali Krishnan, P.B. Sunil Kumar Editors, Springer. 
Redjem-Saad, L., 2008. Simulation numérique des transferts de chaleur turbulents par convection forcée dans des conduites cylindriques et des espaces annulaires. Phd Thesis, Université Paris-Est/Marne-la-Vallée.

Redjem-Saad, L., Ould-Rouiss, M. and Lauriat, G., 2007. Direct numerical simulation of turbulent heat transfer in pipe flows: Effect of Prandtl number. Int. J. Heat and Fluid Flow 28, 847-861.

Ro Kyoungchul and Ryou HongSun, 2012. Development of the modified $k-\epsilon$ turbulence model of power-law fluid for engineering applications. Science China 55, $n^{\circ} 1,276-284$.

Rudman, M., Blackburn, H.M., Graham, L.J.W., and Pullum, L., 2001. Weakly turbulent pipe flow of a power law fluid. Proceedings of the 4th A/Asian Fluid Mechanics Conference, Adelaide, 2001.

Rudman, M., Graham, L.J.W., Blackburn, H.M., and Pullum, L., 2002. Non-Newtonian turbulent and transitional pipe flow. Hydrotransport 15, Banff, 2002.

Rudman M., Blackburn H.M., Graham L.J.W. and Pullum L., 2004. Turbulent pipe flow of shear-thinning fluids. J. Non-Newtonian Fluid Mech. 118, 33-48.

Rudman M. and Blackburn H.M, 2012. Turbulence modification in shear-thinning fluids: preliminary results for power law rheology. $18^{\text {th }}$ Australian Fluid Mechanics Conference. Launceston, Australia.

Thais, L., Tejada-Martinez, A.E., Gatski, T.B., Mompean, G., 2010. Temporal large eddy simulations of turbulent viscoelastic drag reduction flows. Phys. Fluids 22, 013103.

Verzico, R., Orlandi, P. 1996. A fintie-difference scheme for three dimensional incompressible flows in cylindrical coordinates. J.of Comp. Phys. 123, 402-414.

Xu, C., Zhang, Z., den Toonder, J.M.J., Nieuwstadt, F.T.M., 1996. Origin of high kurtosis levels in the viscous sublayer. Direct numerical simulation and experiment. Phys. Fluids 8, 1938-1944.

Zahrai, S., Bark, F.H., Karlsson, R.I., 1995. On anisotropic subgrid modelling. European Journal of Mechanics B/Fluids 144 459-486.

Zang, T., 1991. Numerical simulation of the dynamics of turbulent boundary layers: perspectives of a transition simulator. In Philosophocal Transaction of the Royal Society of London A 336, 95-102. 


\section{FIGURE CAPTIONS}

Fig.1 Laminar axial velocity profiles compared to analytical solution.

Fig.2a Turbulent mean axial velocity profile compared to experiments.

Fig.2b Effect of $n$ on turbulent axial velocity profiles.

Fig.2c Effect of $R e_{s}$ and $n$ on turbulent axial velocity profiles.

Fig.2d Effect of the sgs model and mesh on the axial velocity profiles, $n=0.8$.

Fig.2e Effect of the sgs model and mesh on the axial velocity profiles, $n=1.2$.

Fig.3a Mean normalized viscosity versus mean normalized shear rate: effect of $n$.

Fig.3b Behaviour of the mean normalized viscosity.

Fig.3c Mean normalized viscosity versus normalized shear rate: effect of $n$ for different $R e_{s}$.

Fig.3d Mean normalized viscosity versus shear rate: effect of $R e_{s}$.

Fig.4a RMS of velocity fluctuations compared to experiments for $n=1$.

Fig.4b RMS of velocity fluctuations compared to experiments for $n=0.9$.

Fig.5a Effect of $n$ on RMS of axial velocity fluctuations.

Fig.5b Effect of $n$ on RMS of radial velocity fluctuations.

Fig.5c Effect of $n$ on RMS of azimuthal velocity fluctuations.

Fig.6a Effect of $R e_{s}$ on RMS of axial velocity fluctuations.

Fig.6b Effect of $R e_{s}$ on RMS of radial velocity fluctuations.

Fig.6c Effect of $R e_{s}$ on RMS of azimuthal velocity fluctuations.

Fig.7a Effect of $n$ on Reynolds shear stress.

Fig.7b Effect of $R e_{s}$ on Reynolds shear stress.

Fig.8 Cross-correlation coefficient.

Fig.9 Peak position of RMS velocity fluctuations and kinetic energy.

Fig.10 Skewness of axial velocity fluctuations: (a) effect of $n$, (b) effect of $R e_{s}$.

Fig.11 Flatness of velocity fluctuations, effect of $n$ : (a) $v_{z}^{\prime}$, (b) $v_{r}^{\prime}$, (c) $v_{\theta}^{\prime}$.

Fig.12 Flatness of radial velocity fluctuations, effect of $R e_{s}$.

Fig.13 Contours of resolved axial velocity field at $y^{+} \simeq 15$ : (a) $n=0.5$, (b) $n=0.75$, (c) $n=1.0$, (b) $n=1.2$.

Fig.14 Contours of resolved axial velocity field in $r-\theta$-plane: (a) $n=0.5$, (b) $n=0.75$, (c) $n=1.0$, (b) $n=1.2$. 
TABLE I: Parameters of present LES simulations and mean flow quantities for $R e_{s}=4000$

\begin{tabular}{llllllll}
\hline Parameters & $\mathrm{n}=0.5$ & $\mathrm{n}=0.69$ & $\mathrm{n}=0.75$ & $\mathrm{n}=0.8$ & $\mathrm{n}=1.0$ & $\mathrm{n}=1.2$ & $\mathrm{n}=1.4$ \\
$\Delta z^{+}$ & 33.84 & 38.88 & 41.14 & 43.01 & 50.08 & 59.85 & 75.23 \\
$(r \Delta \theta)^{+}$ & 10.63 & 12.21 & 12.85 & 13.51 & 15.73 & 18.80 & 23.63 \\
$\Delta r_{\text {min }}^{+}$ & 0.084 & 0.059 & 0.054 & 0.051 & 0.040 & 0.034 & 0.030 \\
$\Delta r_{\text {max }}^{+}$ & 18.57 & 12.98 & 12.00 & 11.27 & 8.87 & 7.56 & 6.69 \\
$U_{b} / U_{c l}$ & 0.542 & 0.545 & 0.533 & 0.524 & 0.494 & 0.502 & 0.544 \\
$U_{c} / U_{c l}$ & 0.757 & 0.701 & 0.687 & 0.677 & 0.647 & 0.662 & 0.725 \\
$U_{\tau} / U_{c l}$ & 0.027 & 0.032 & 0.033 & 0.034 & 0.036 & 0.039 & 0.045 \\
$<\dot{\gamma}_{d, w}>$ & 2.794 & 6.730 & 5.902 & 5.942 & 5.377 & 5.366 & 5.437 \\
$<\eta_{d, w}>\times 10^{4}$ & 1.49 & 1.38 & 1.60 & 1.75 & 2.50 & 3.50 & 4.92 \\
$R e_{c r}$ & 2464 & 2280.3 & 2236.7 & 2204.2 & 2100 & 2024.6 & 1967.5 \\
$R e_{M R}$ & 5302 & 5033 & 4870 & 4754 & 4449 & 4512 & 4731 \\
$R e_{w}$ & 10211 & 8011 & 7042 & 6359 & 4449 & 3506 & 2920 \\
$R e_{\tau}=U_{\tau} R / \nu_{w}$ & 110 & 126.4 & 133.72 & 139.81 & 162.77 & 194.52 & 244.51 \\
$f_{D M} \times 10^{+3}$ & 5.82 & 7.32 & 7.79 & 8.18 & 9.58 & 10.53 & 11.00 \\
$f \times 10^{3}$ & 6.35 & 8.37 & 8.85 & 9.24 & 10.46 & 12.54 & 15.69 \\
$f_{G} \times 10^{3}$ & 6.13 & 7.58 & 8.06 & 8.44 & 9.87 & - & - \\
$y_{1}^{+}$ & 0.0342 & 0.0251 & 0.0231 & 0.0217 & 0.0171 & 0.0145 & 0.0129
\end{tabular}


TABLE II: Parameters and mean flow quantities for $R e_{s}=8000$

\begin{tabular}{llll}
\hline Parameters & $\mathrm{n}=0.8$ & $\mathrm{n}=1$ & $\mathrm{n}=1.2$ \\
$\Delta z^{+}$ & 65.56 & 78.97 & 99.37 \\
$(r \Delta \theta)^{+}$ & 21.17 & 24.81 & 31.22 \\
$\Delta r_{\min }^{+}$ & 0.087 & 0.063 & 0.053 \\
$\Delta r_{\text {max }}^{+}$ & 19.13 & 14.01 & 11.65 \\
$U_{b} / U_{c L}$ & 0.525 & 0.495 & 0.529 \\
$U_{c} / U_{c L}$ & 0.640 & 0.617 & 0.663 \\
$U_{\tau} / U_{c L}$ & 0.029 & 0.032 & 0.037 \\
$<\dot{\gamma}_{d, w}>$ & 9.470 & 7.759 & 7.931 \\
$<\eta_{d, w}>\times 10^{4}$ & 0.797 & 1.250 & 1.891 \\
$R e_{c r}$ & 2204.2 & 2100. & 2024.6 \\
$R e_{M R}$ & 8485 & 7928 & 8368 \\
$R e_{w}$ & 12291 & 7928 & 6109 \\
$R e_{\tau}=U_{\tau} R / \nu_{w}$ & 219.1 & 256.7 & 323.0 \\
$f_{D M} \times 10^{+3}$ & 7.02 & 8.29 & 9.0 \\
$f \times 10^{3}$ & 7.18 & 8.23 & 10.94 \\
$f_{G} \times 10^{3}$ & 7.15 & 8.36 & - \\
$y_{1}^{+}$ & 0.0369 & 0.0270 & 0.0225
\end{tabular}


TABLE III: Parameters and mean flow quantities for $R e_{s}=12000$

\begin{tabular}{llll}
\hline Parameters & $\mathrm{n}=0.8$ & $\mathrm{n}=1$ & $\mathrm{n}=1.2$ \\
$\Delta z^{+}$ & 92.68 & 109.65 & 139.07 \\
$(r \Delta \theta)^{+}$ & 29.11 & 34.44 & 43.69 \\
$\Delta r_{\text {min }}^{+}$ & 0.127 & 0.063 & 0.071 \\
$\Delta r_{\text {max }}^{+}$ & 27.87 & 19.44 & 15.61 \\
$U_{b} / U_{c l}$ & 0.526 & 0.496 & 0.530 \\
$U_{c} / U_{c l}$ & 0.626 & 0.603 & 0.652 \\
$U_{\tau} / U_{c l}$ & 0.027 & 0.029 & 0.034 \\
$<\dot{\gamma}_{d, w}>$ & 12.278 & 9.774 & 9.609 \\
$<\eta_{d, w}>\times 10^{4}$ & 0.504 & 0.833 & 1.310 \\
$R e_{c r}$ & 2204.2 & 2100. & 2024.6 \\
$R e_{M R}$ & 12745 & 11908 & 12579 \\
$R e_{w}$ & 19556 & 11908 & 8788 \\
$R e_{\tau}=U_{\tau} R / \nu_{w}$ & 301.2 & 356.3 & 452.0 \\
$f_{D M} \times 10^{+3}$ & 6.31 & 7.49 & 8.22 \\
$f \times 10^{3}$ & 6.03 & 7.05 & 9.52 \\
$f_{G} \times 10^{3}$ & 6.36 & 7.44 & - \\
$y_{1}^{+}$ & 0.0538 & 0.0375 & 0.0301
\end{tabular}




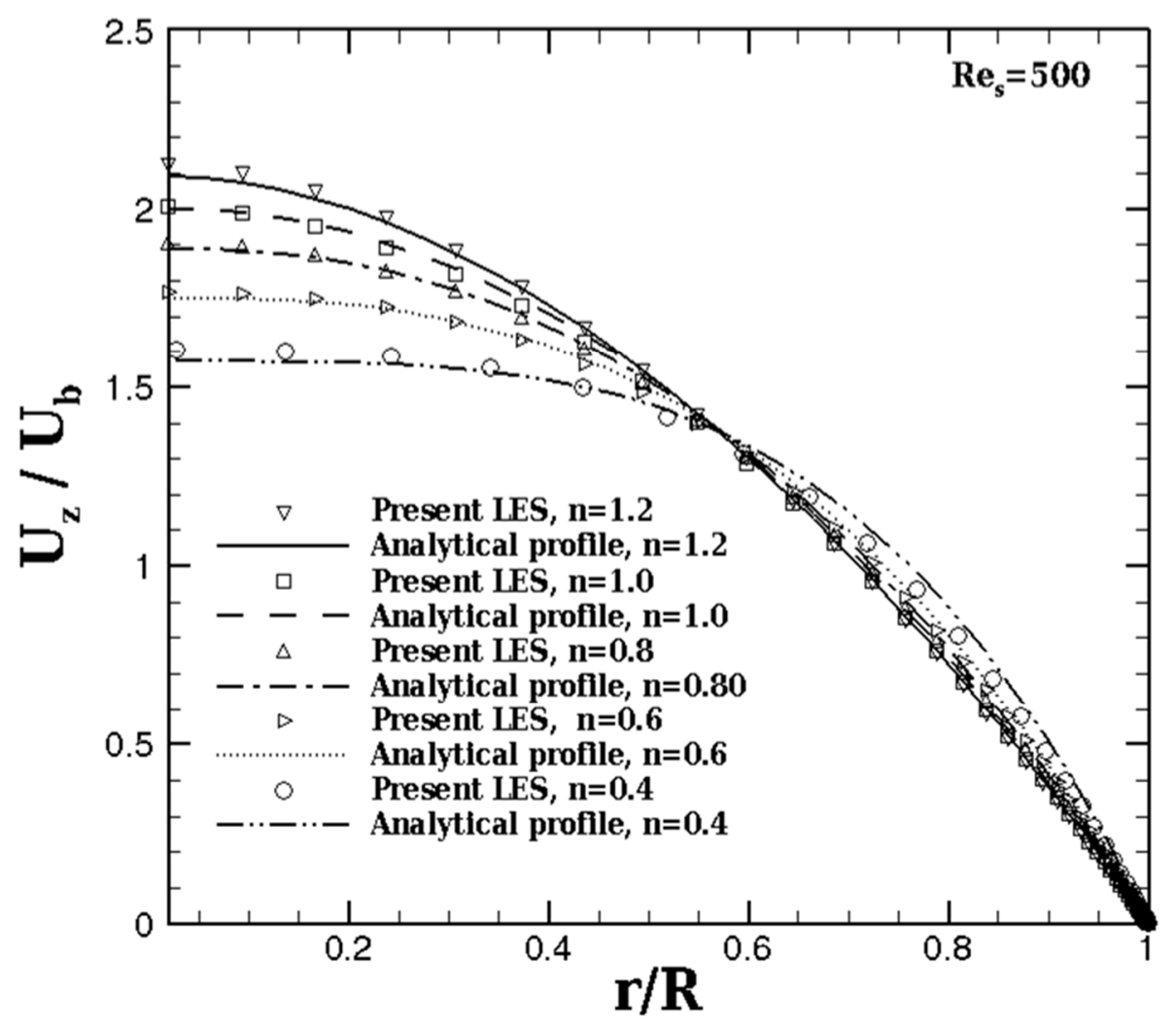

Fig.1 Laminar axial velocity profiles compared to analytical solution.

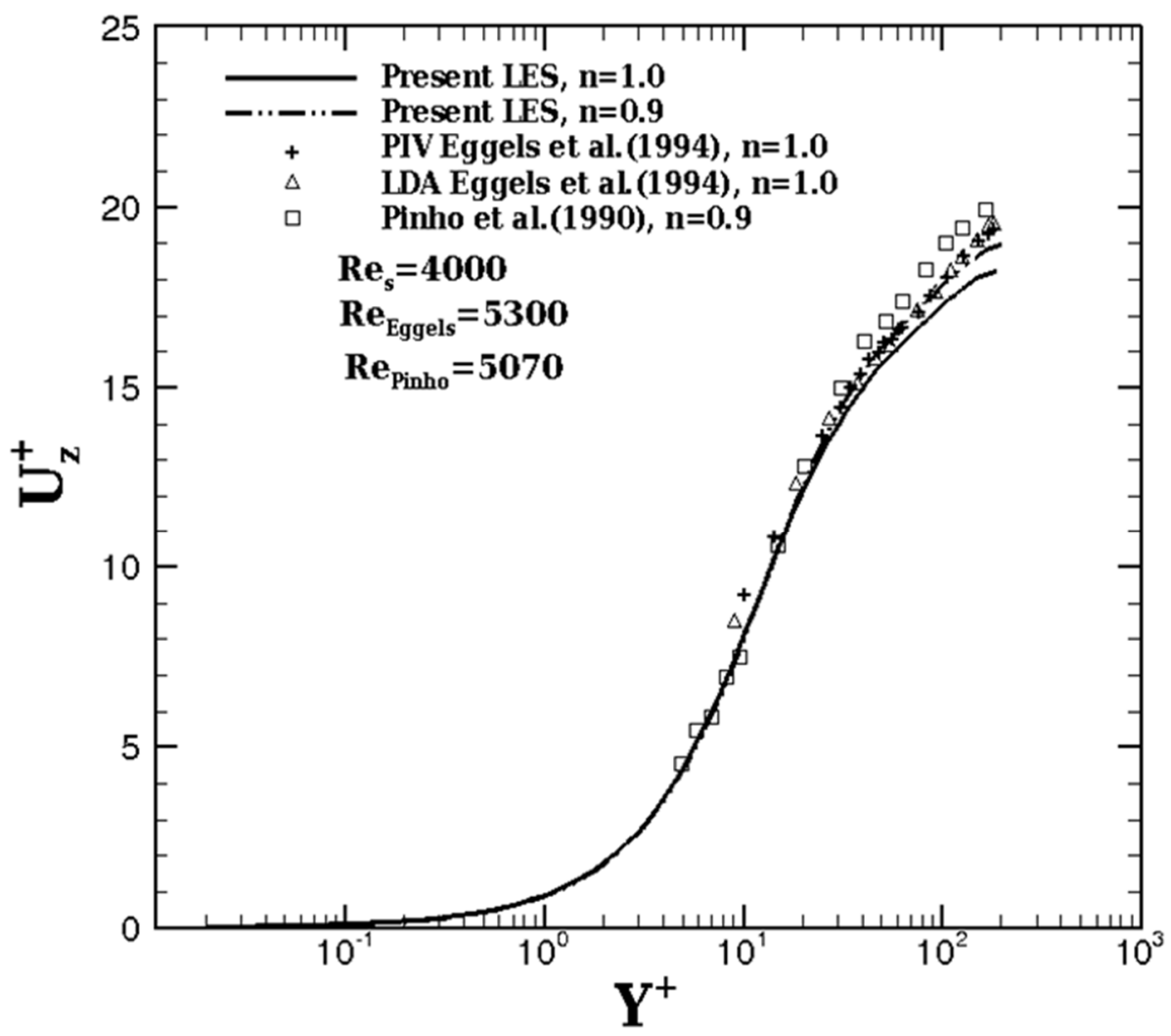

Fig.2a Turbulent mean axial velocity profile compared to experiments. 


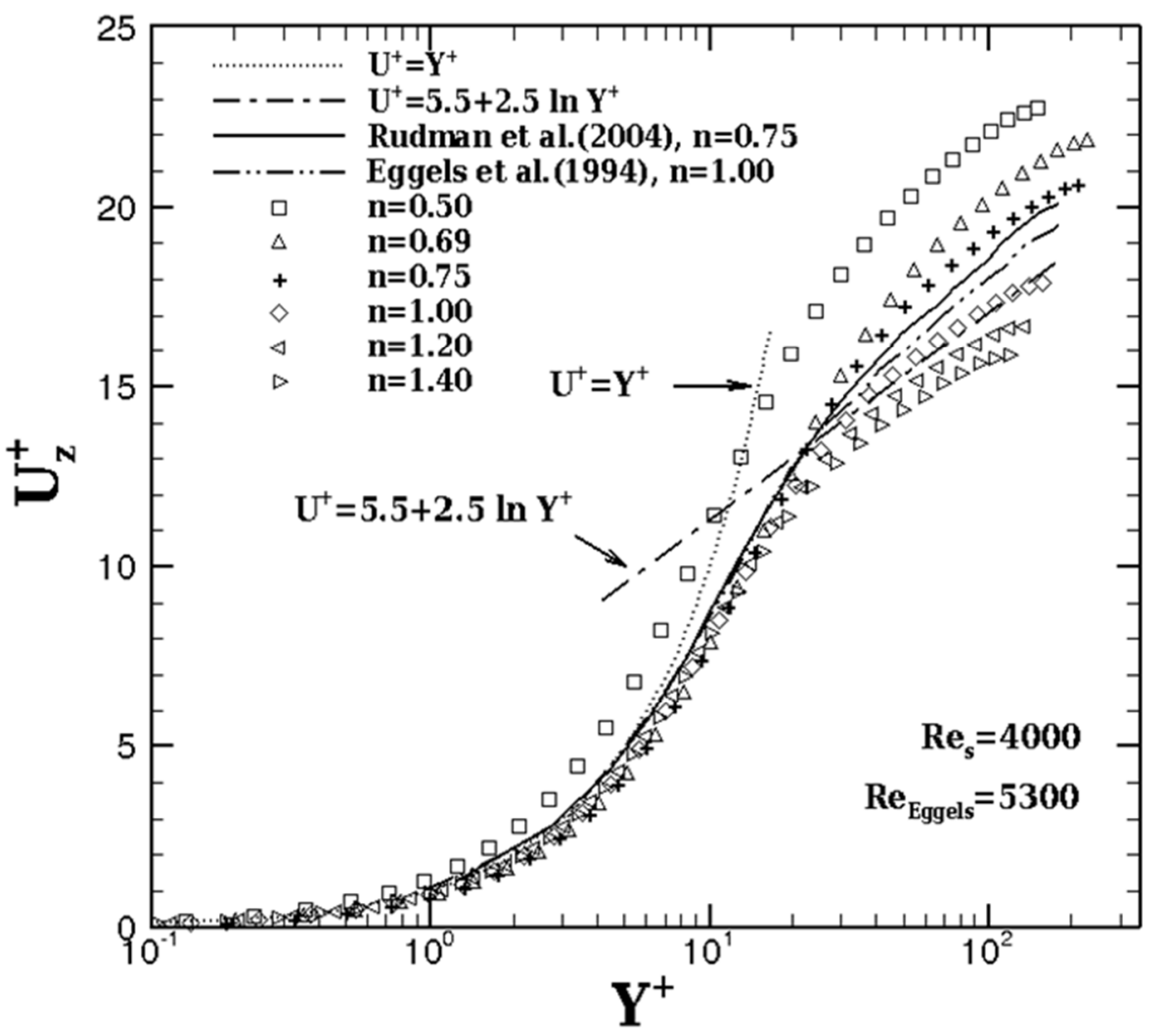

Fig.2b Effect of $\mathrm{n}$ on turbulent axial velocity profiles.

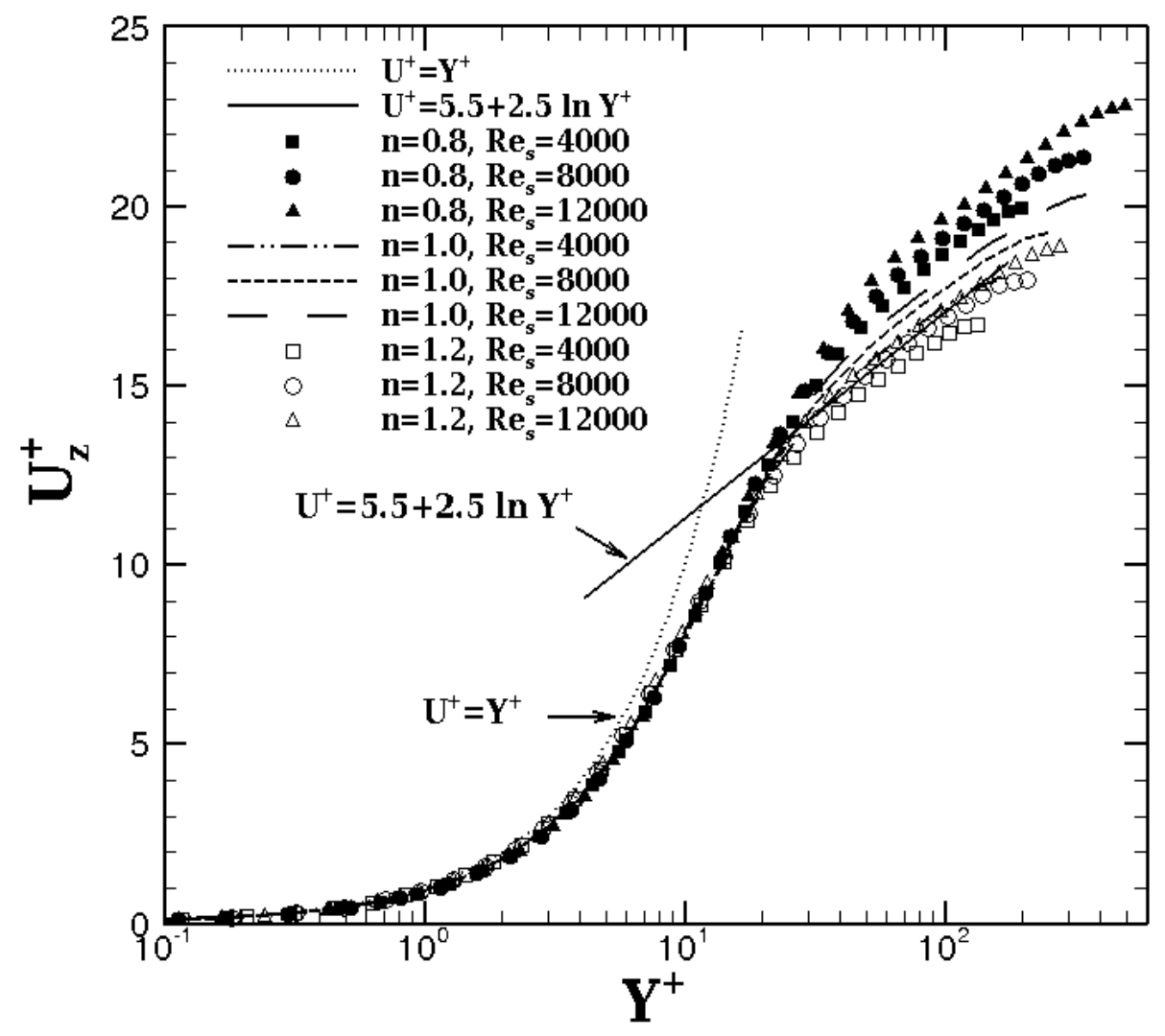

Fig.2c Effect of $\operatorname{Re}_{\mathrm{s}}$ on turbulent axial velocity profiles. 


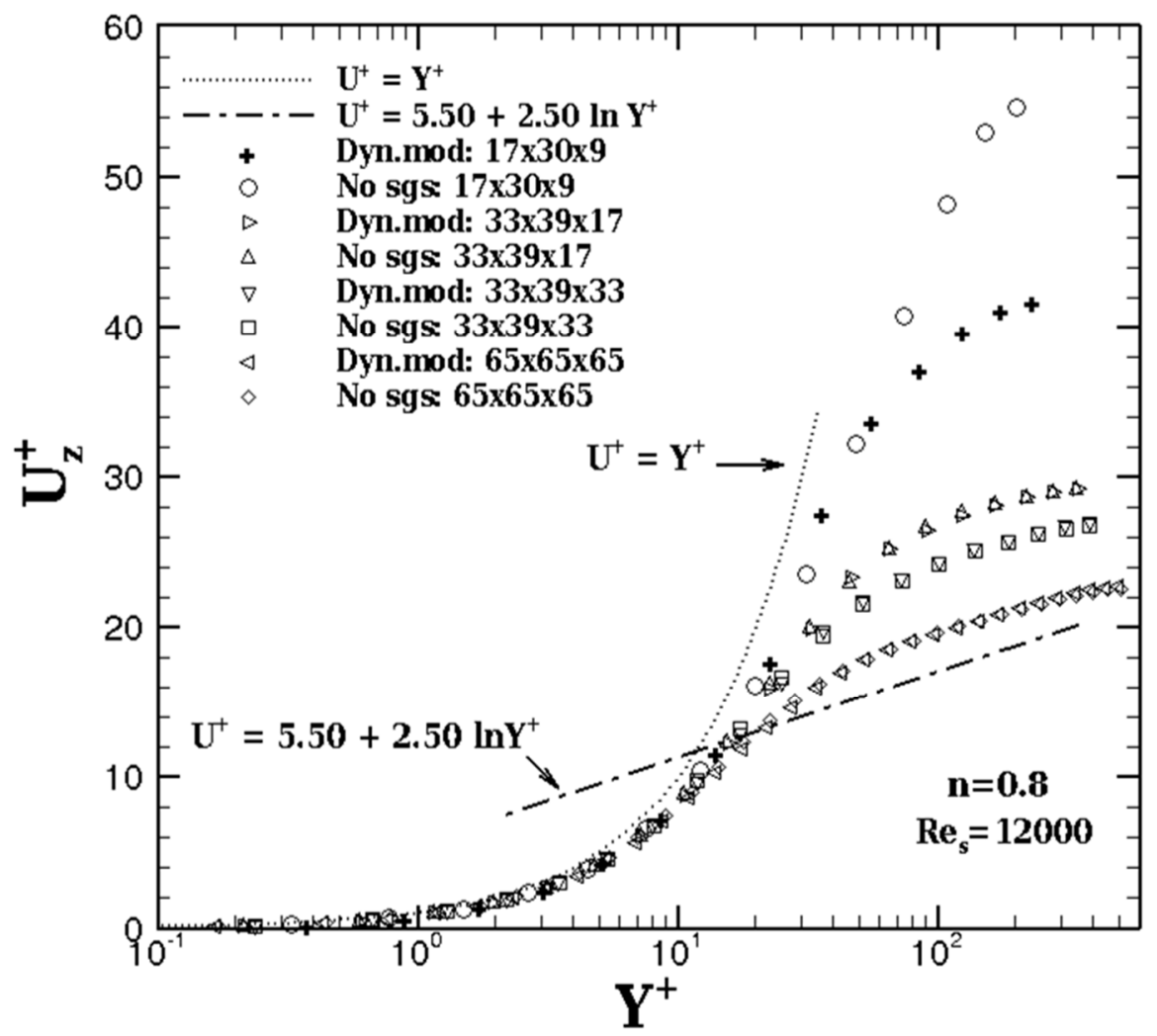

Fig. $2 \mathrm{~d}$ Effect of the sgs model and mesh on the axial velocity profile, $\mathrm{n}=0.8$.

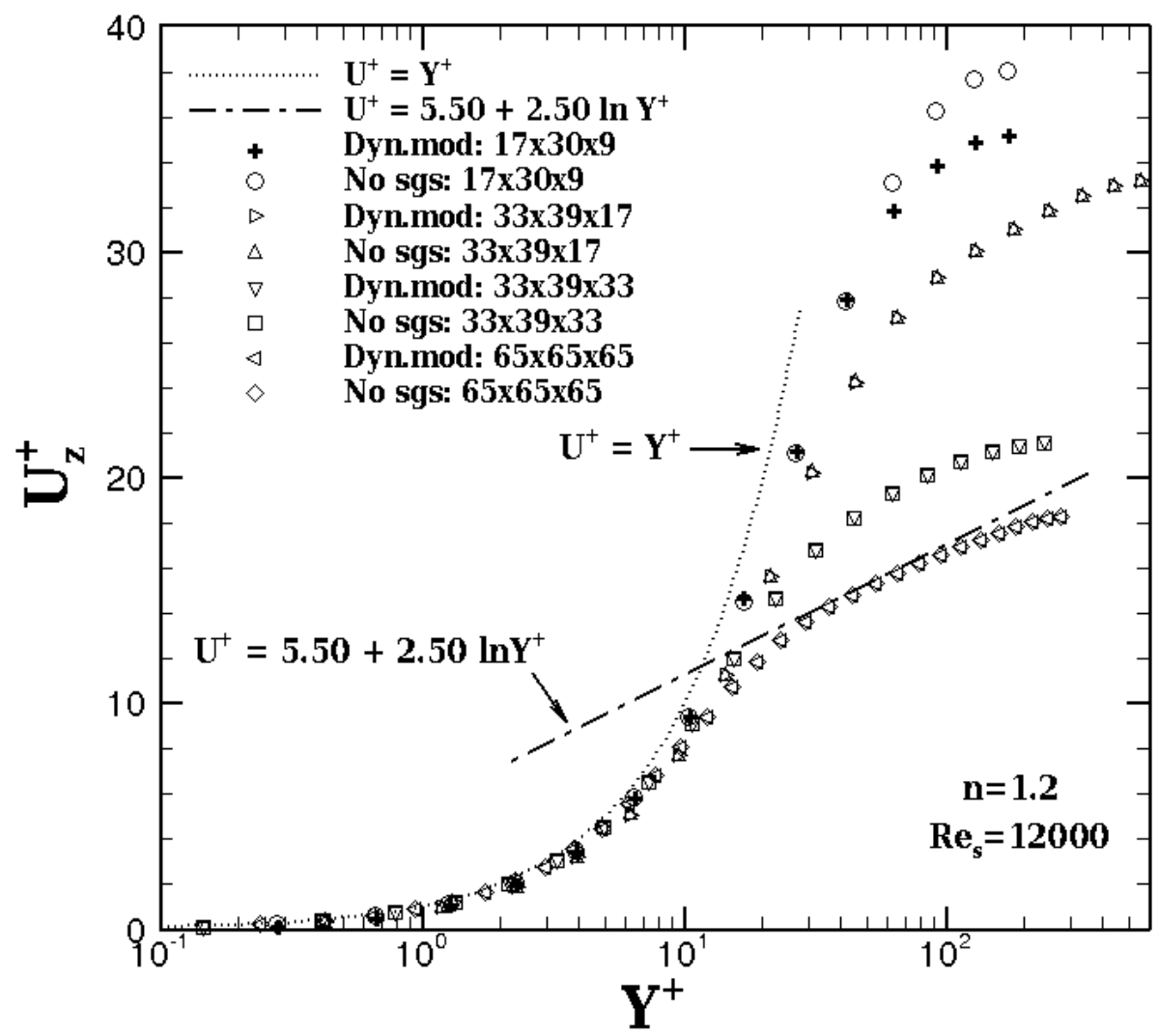

Fig.2e Effect of the sgs model and mesh on the axial velocity profile, $\mathrm{n}=1.2$. 


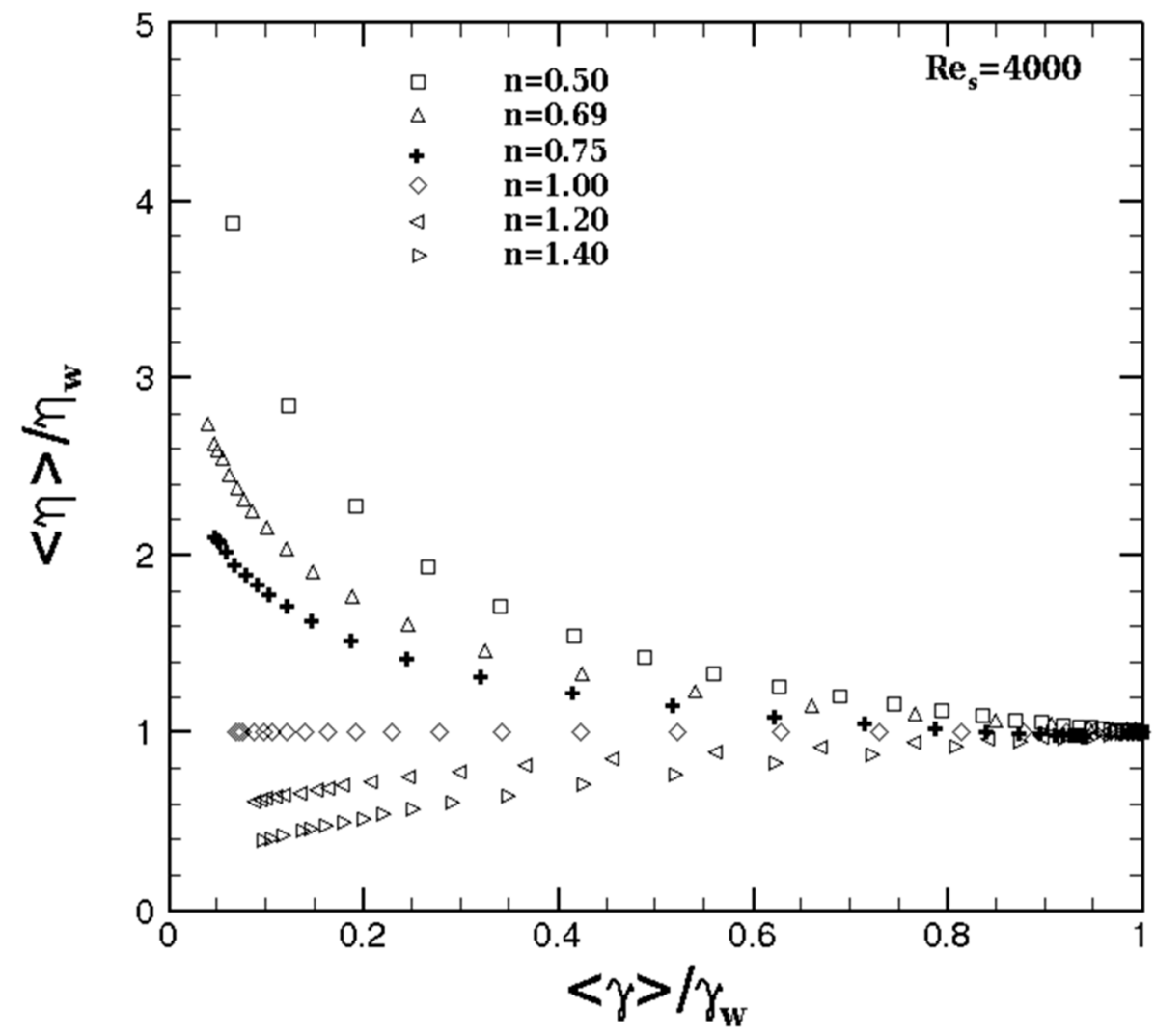

Fig.3a Mean normalized viscosity versus mean normalized shear rate: effect of $n$.

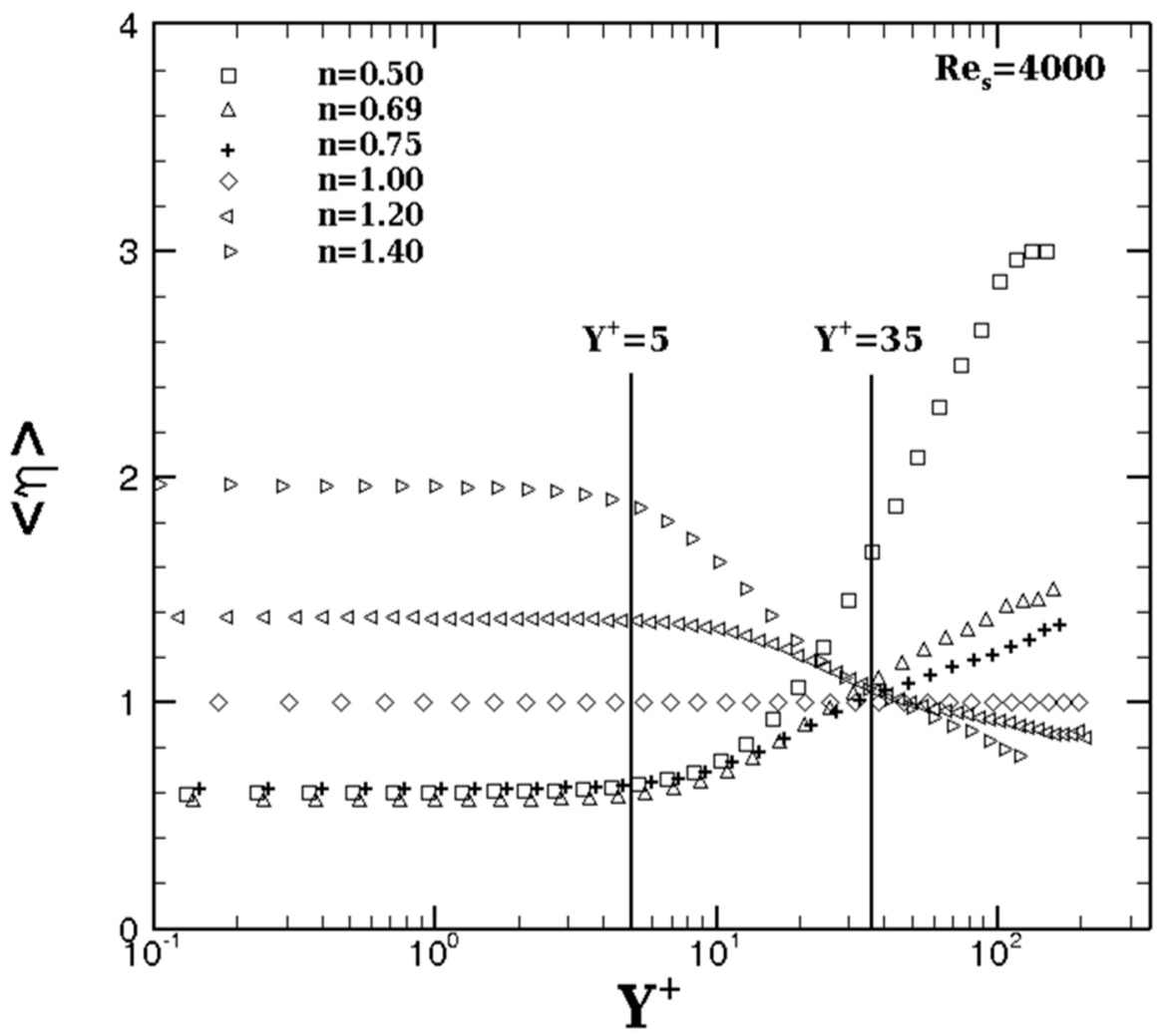

Fig.3b Behaviour of mean dimensionless viscosity 


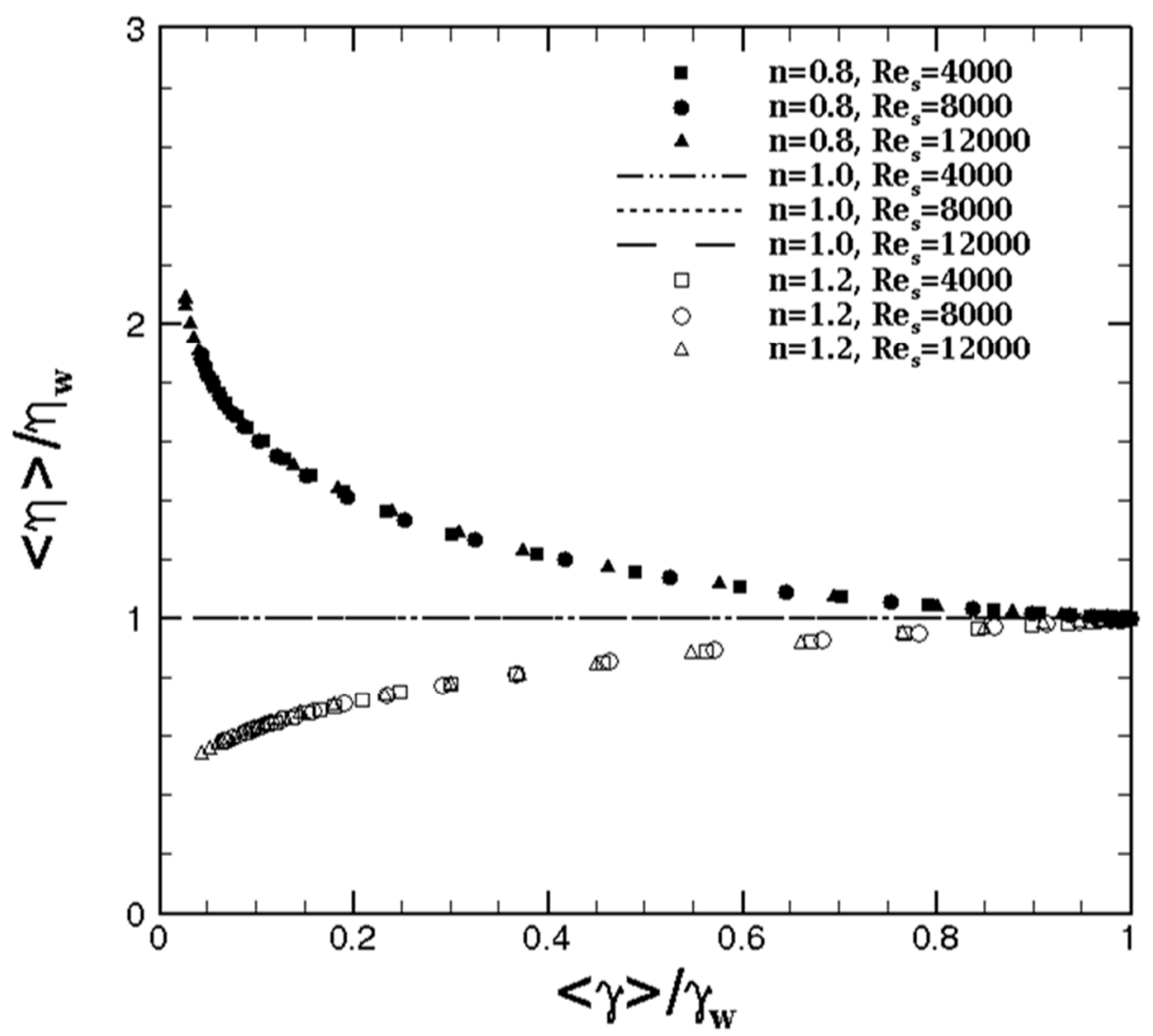

Fig.3c Normalized viscosity versus Normalized shear rate: effect of $\mathrm{n}$ for different $\mathrm{Re}_{\mathrm{s}}$

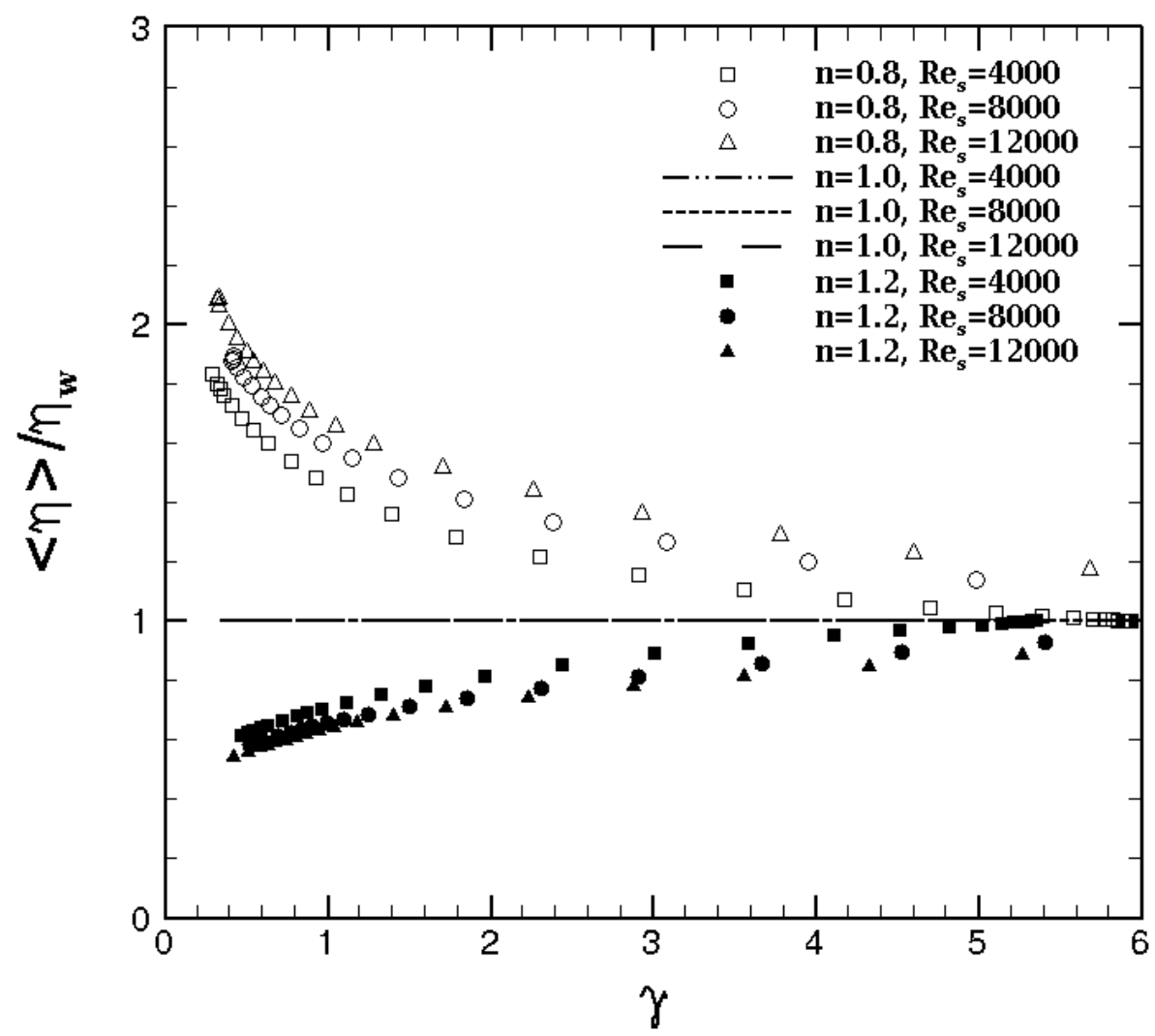

Fig.3d Normalized viscosity versus shear rate: effect of $\operatorname{Re}_{\mathrm{s}}$ 


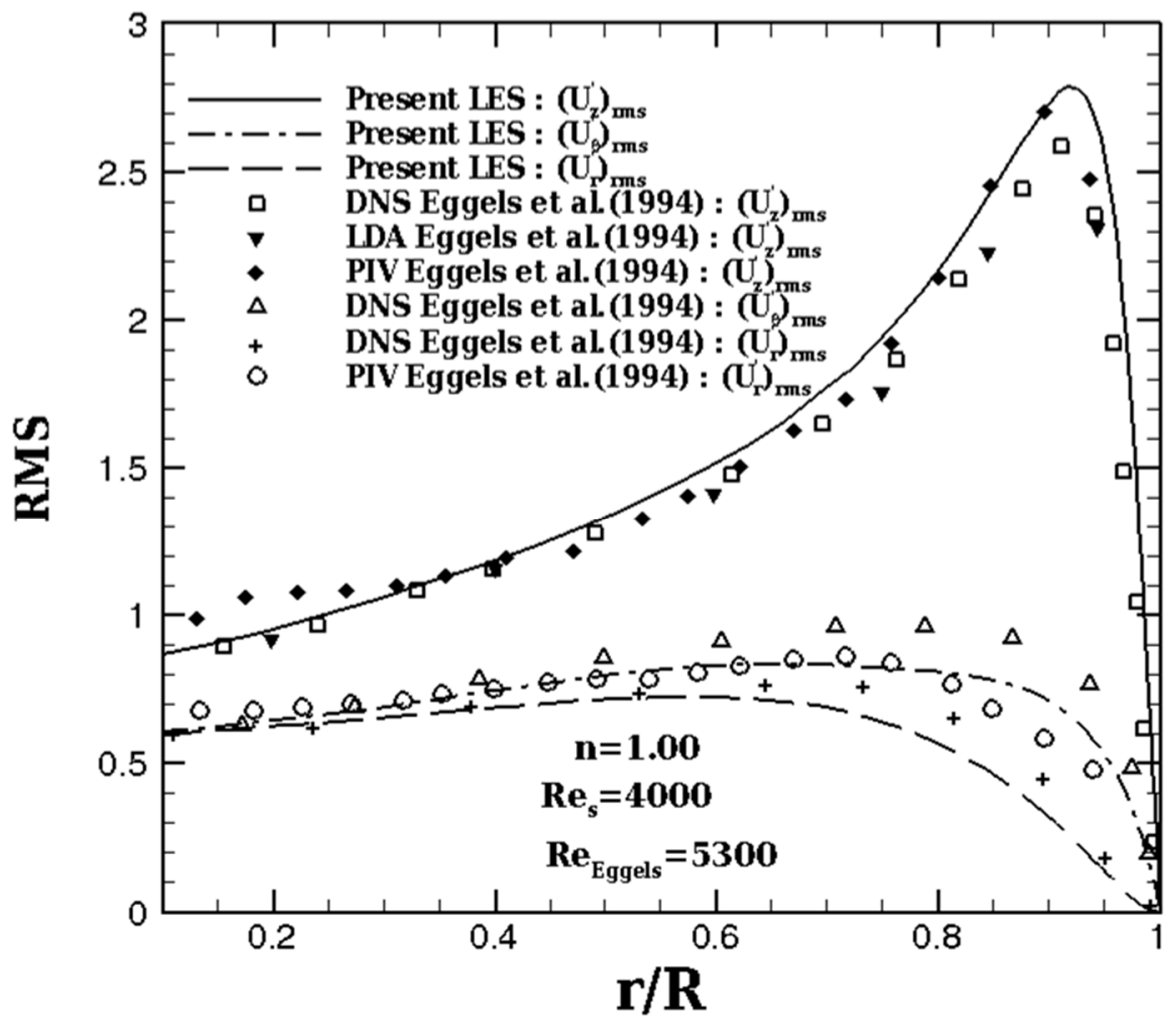

Fig.4a RMS of velocity fluctuations compared to experiments for $\mathrm{n}=1$.

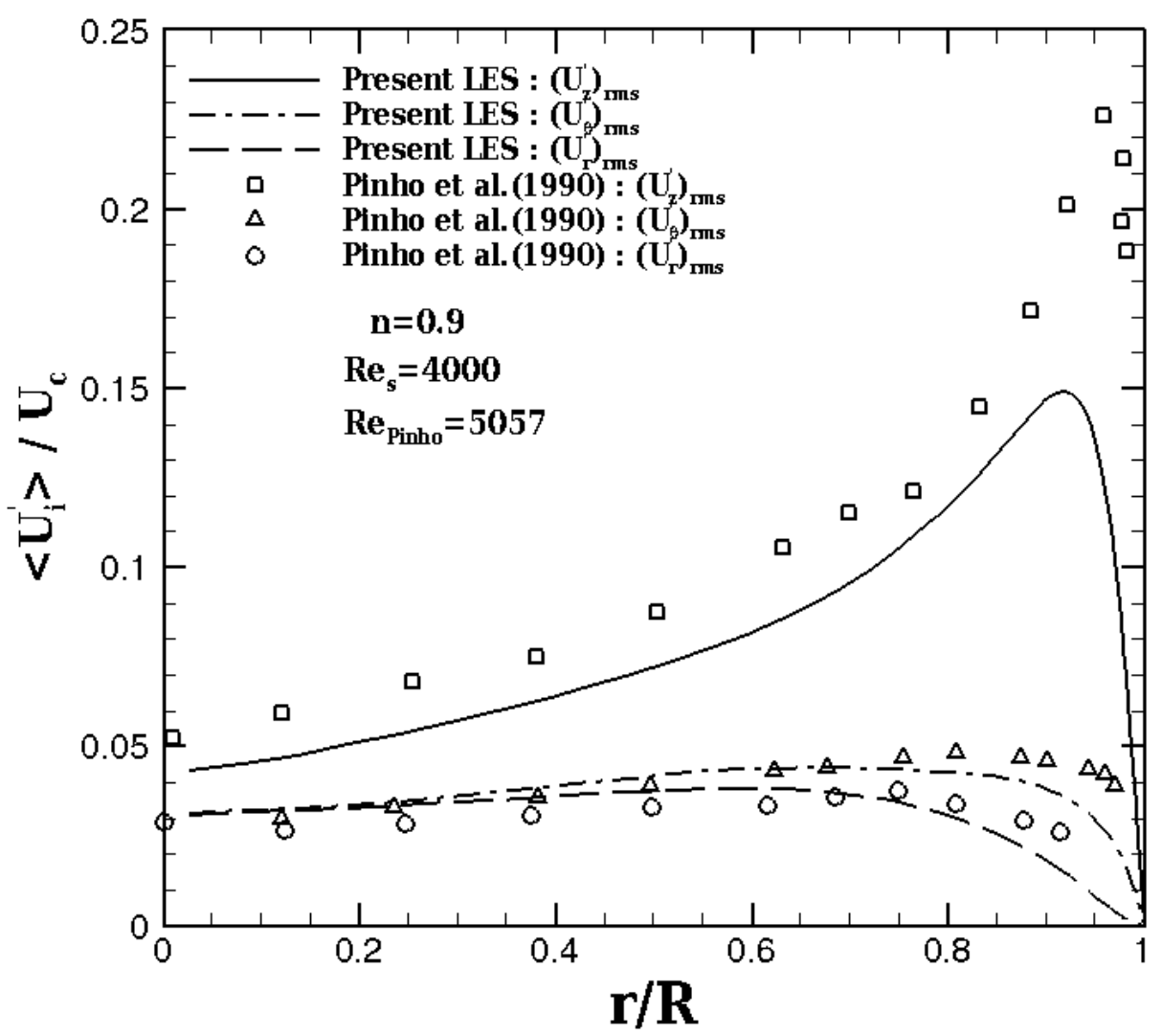

Fig.4b RMS of velocity fluctuations compared to experiments for $n=0.9$. 


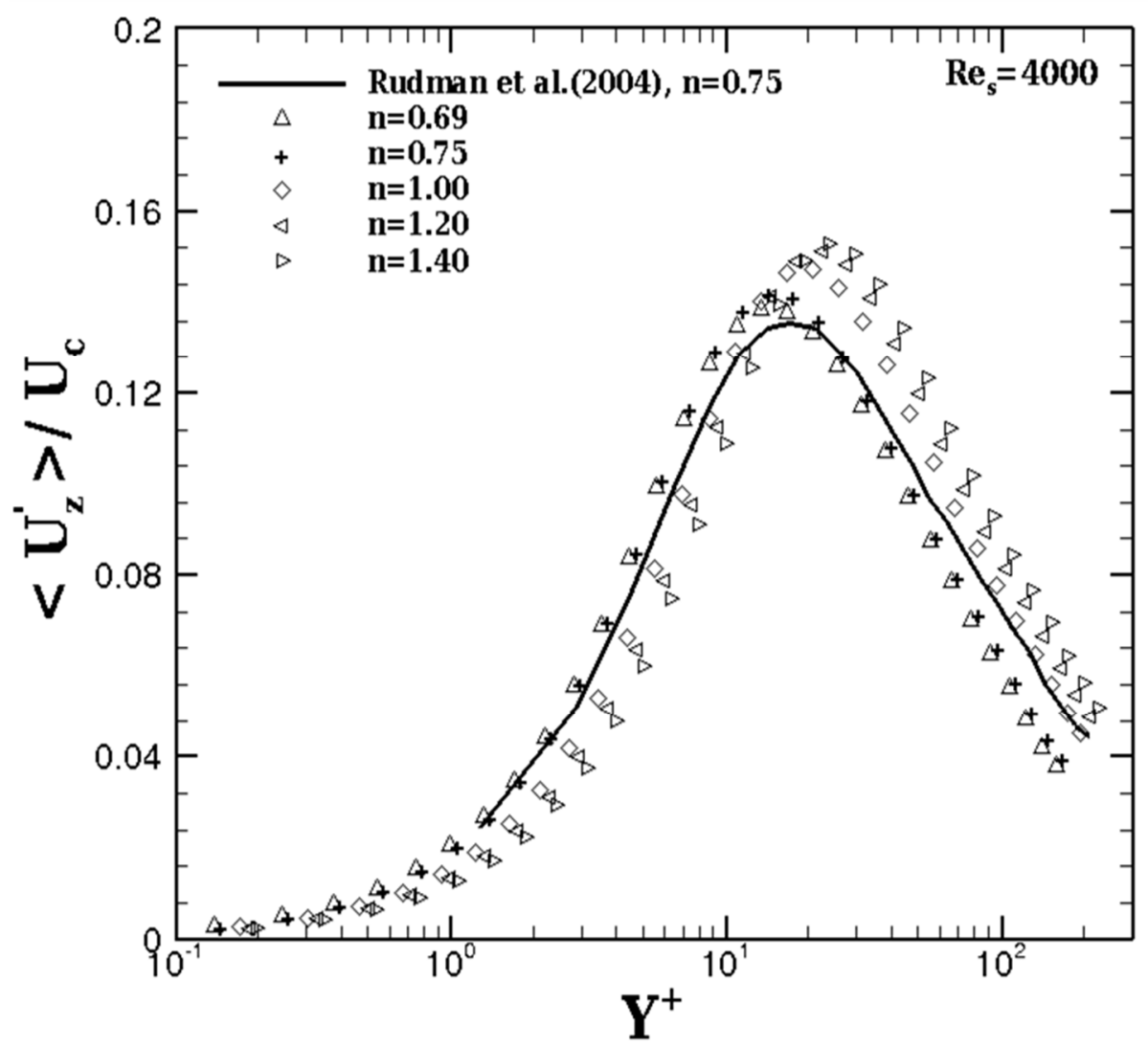

Fig.5a Effect of $\mathrm{n}$ on RMS of axial velocity fluctuations.

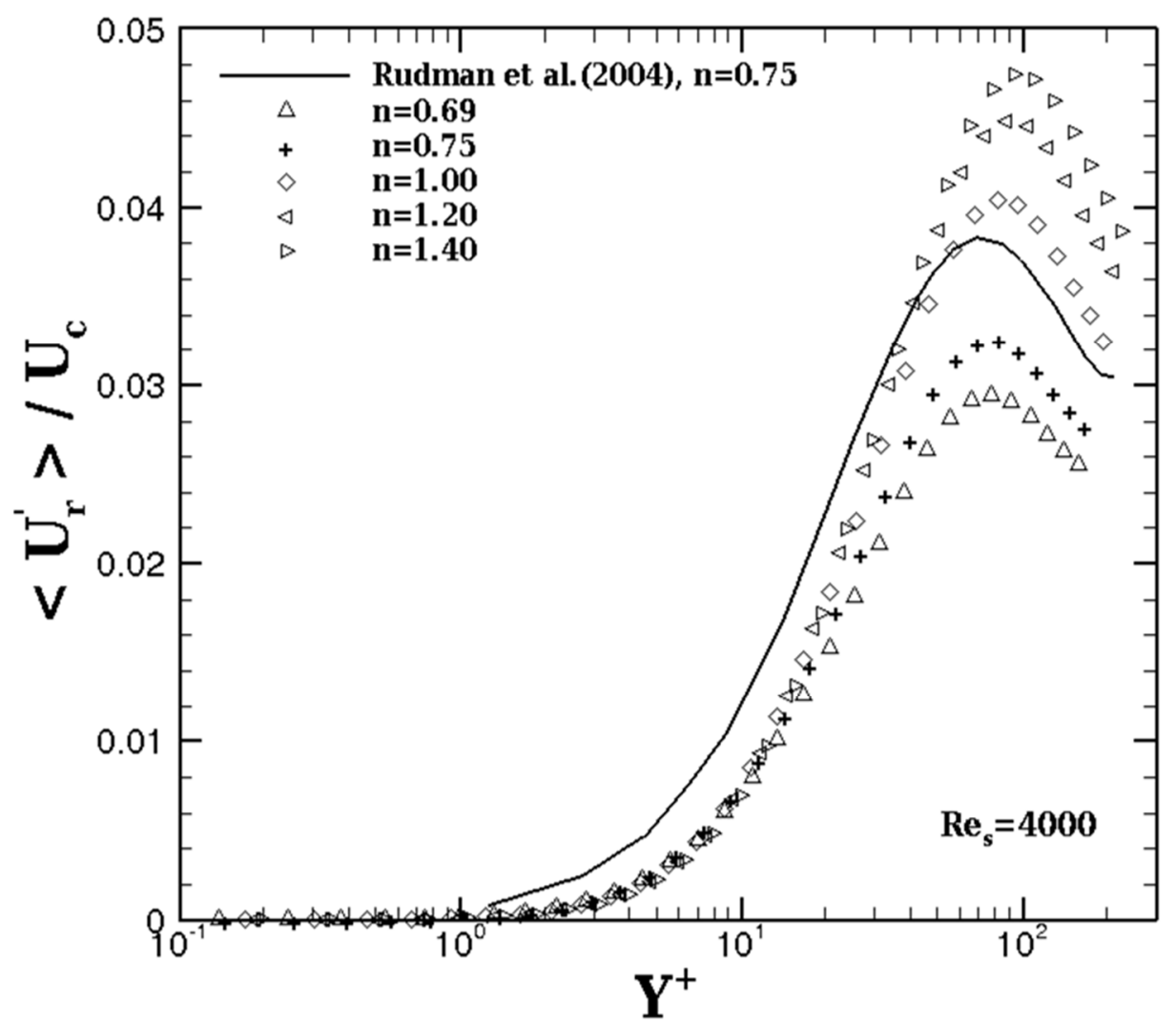

Fig.5b Effect of $\mathrm{n}$ on RMS of radial velocity fluctuations. 


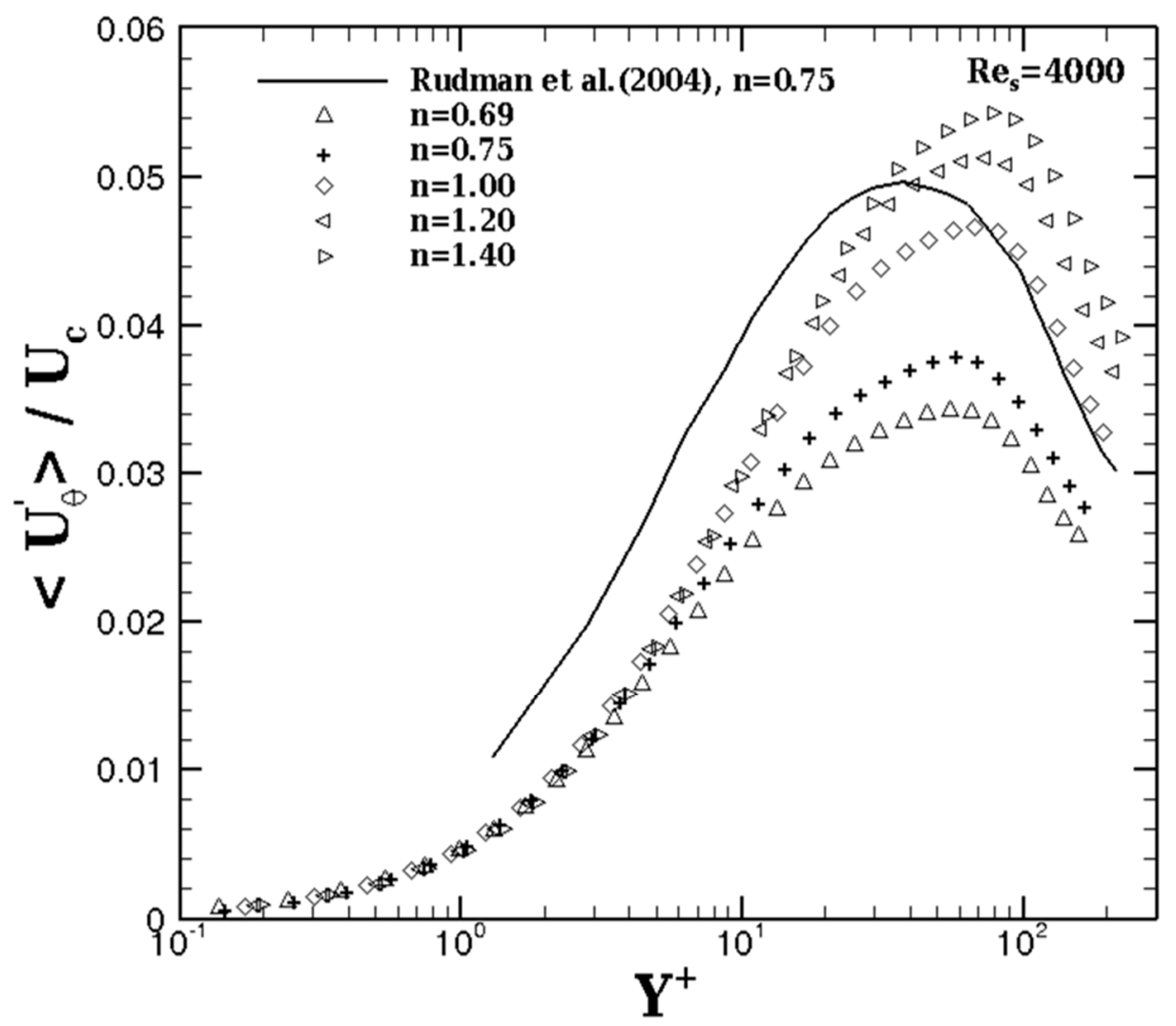

Fig.5c Effect of $\mathrm{n}$ on RMS of azimuthal velocity fluctuations.

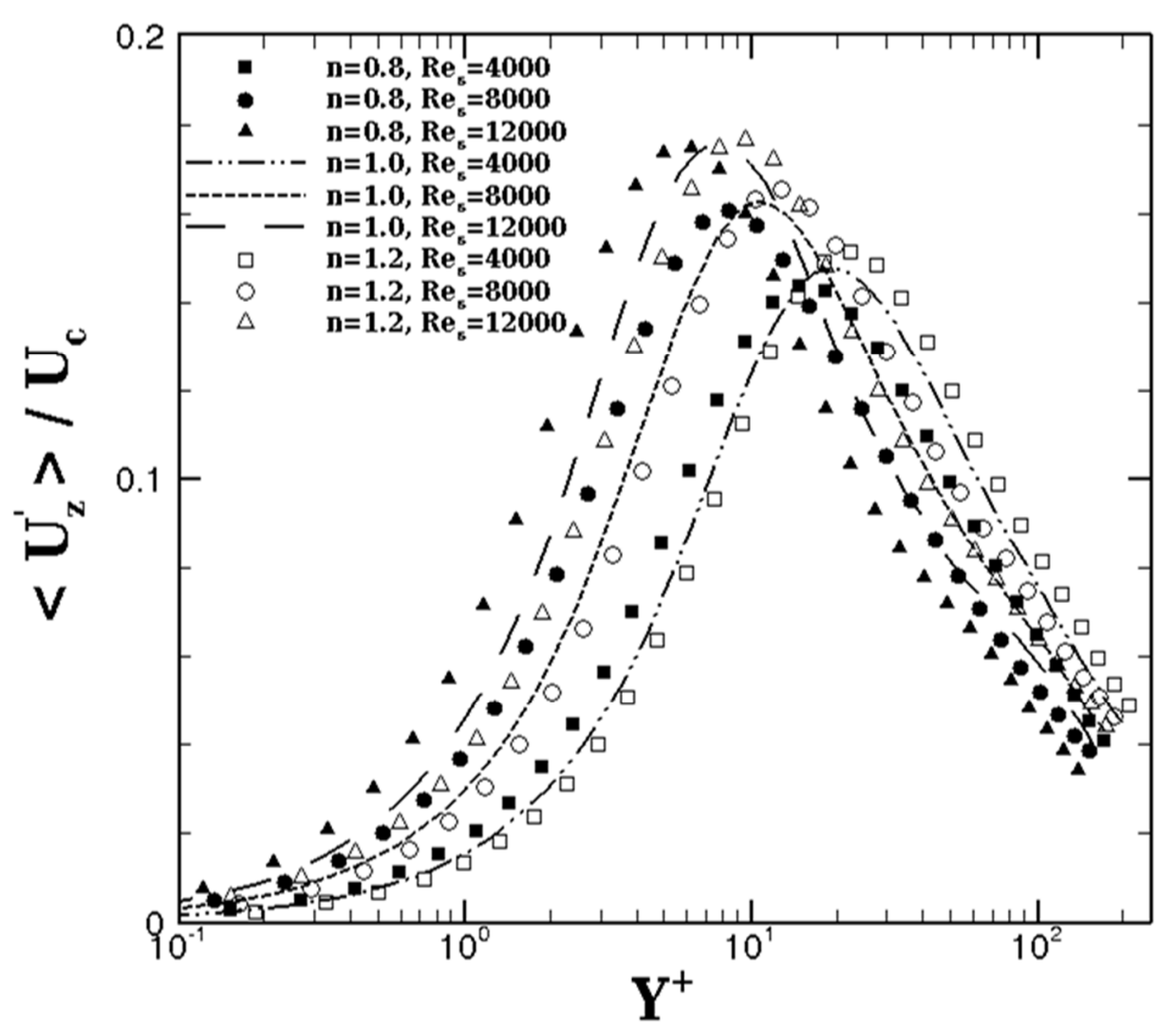

Fig.6a Effect of $\operatorname{Re}_{\mathrm{s}}$ on RMS of axial velocity fluctuations. 


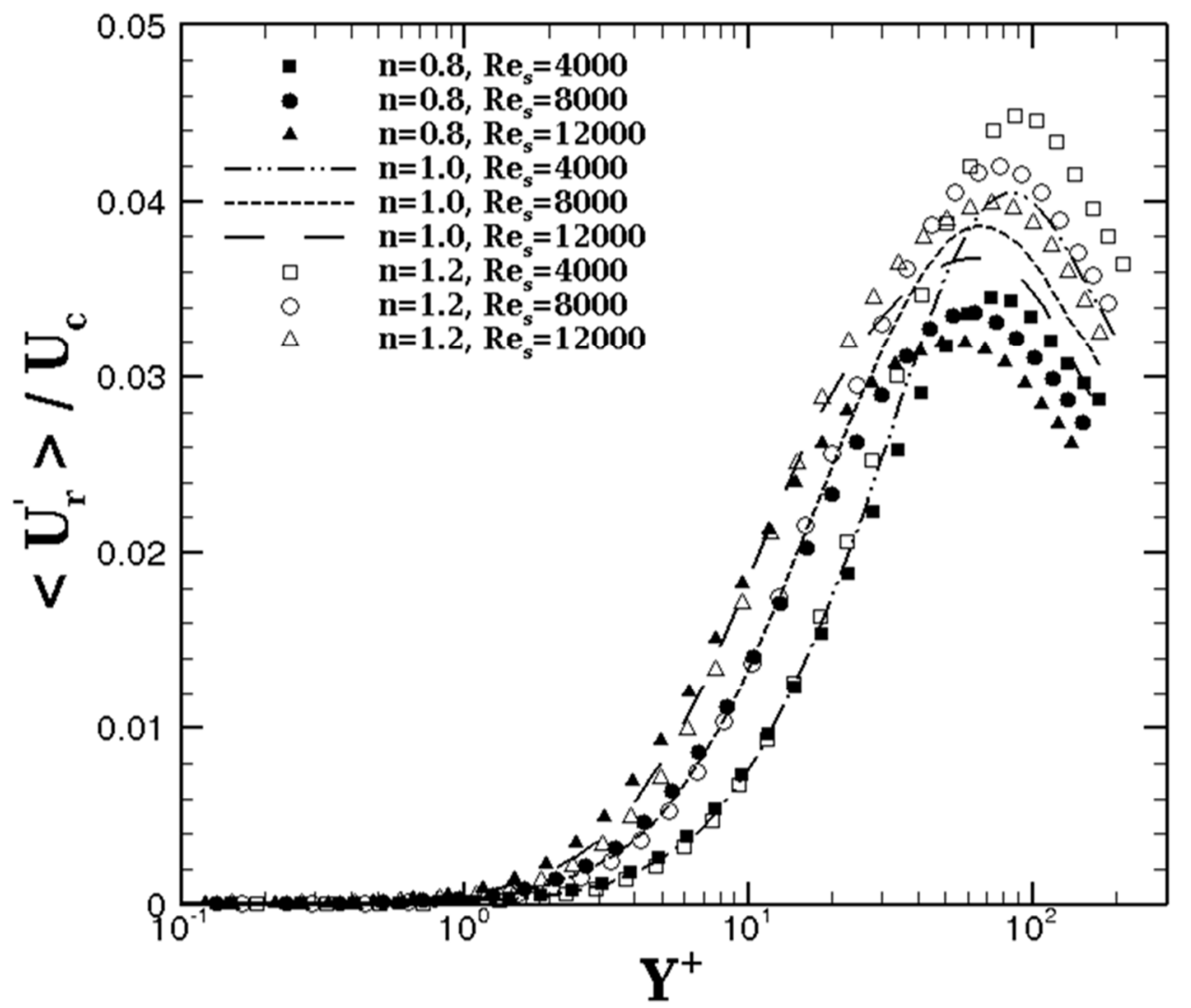

Fig.6b Effect of $\mathrm{Re}_{\mathrm{s}}$ on RMS of radial velocity fluctuations.

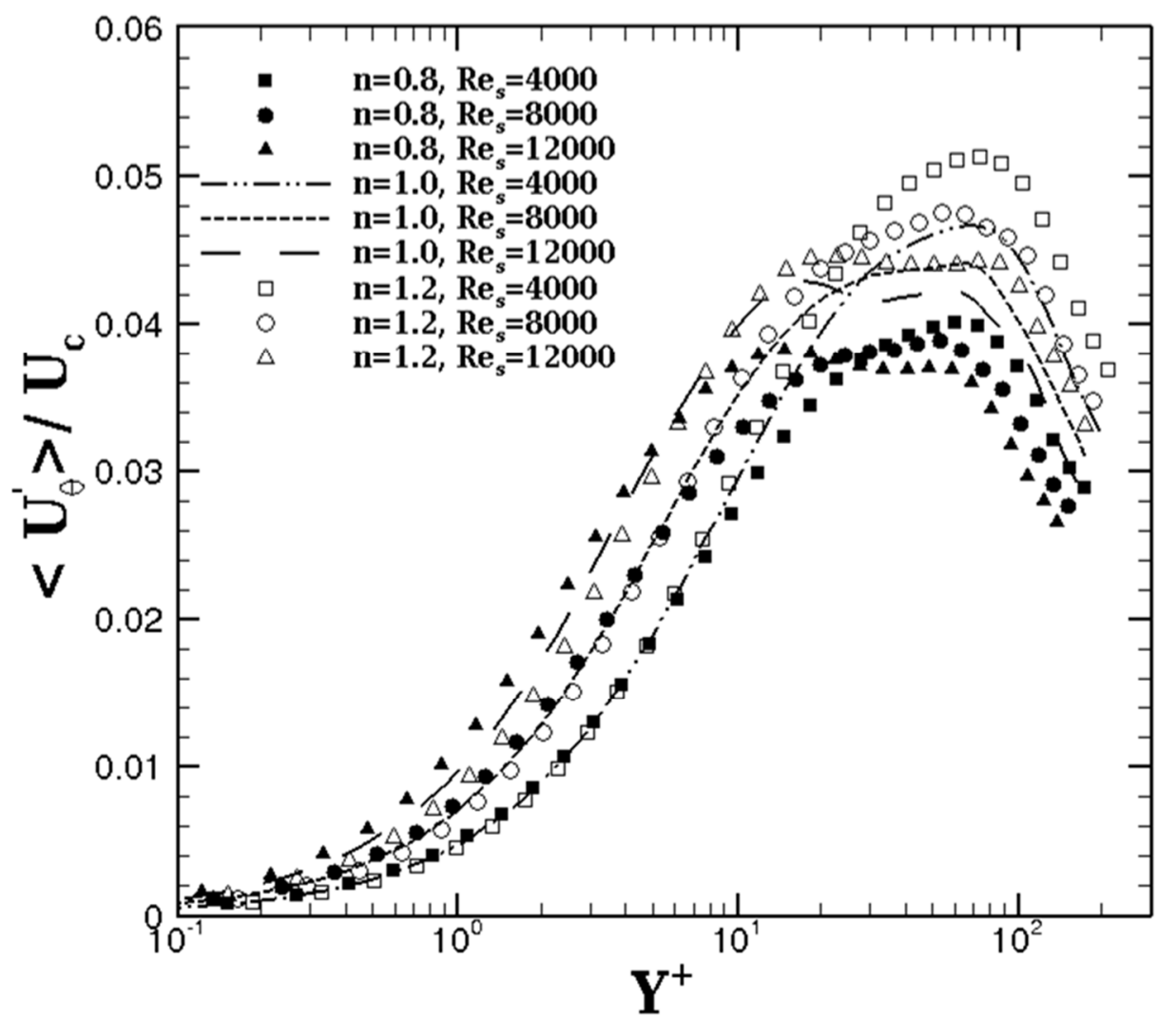

Fig.6c Effect of $\operatorname{Re}_{\mathrm{s}}$ on RMS of azimuthal velocity fluctuations. 


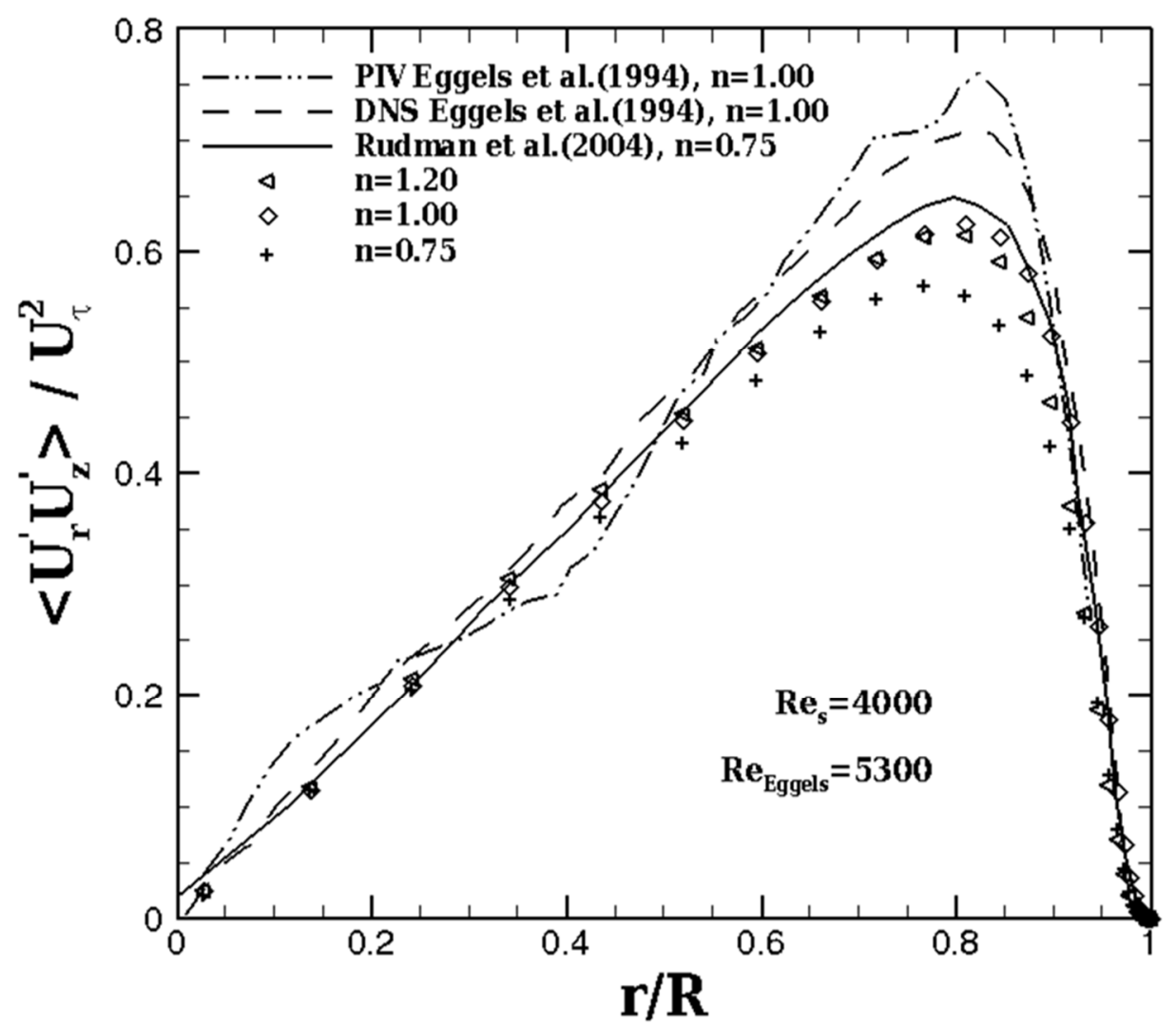

Fig.7a Effect of $\mathrm{n}$ on Reynolds shear stress.

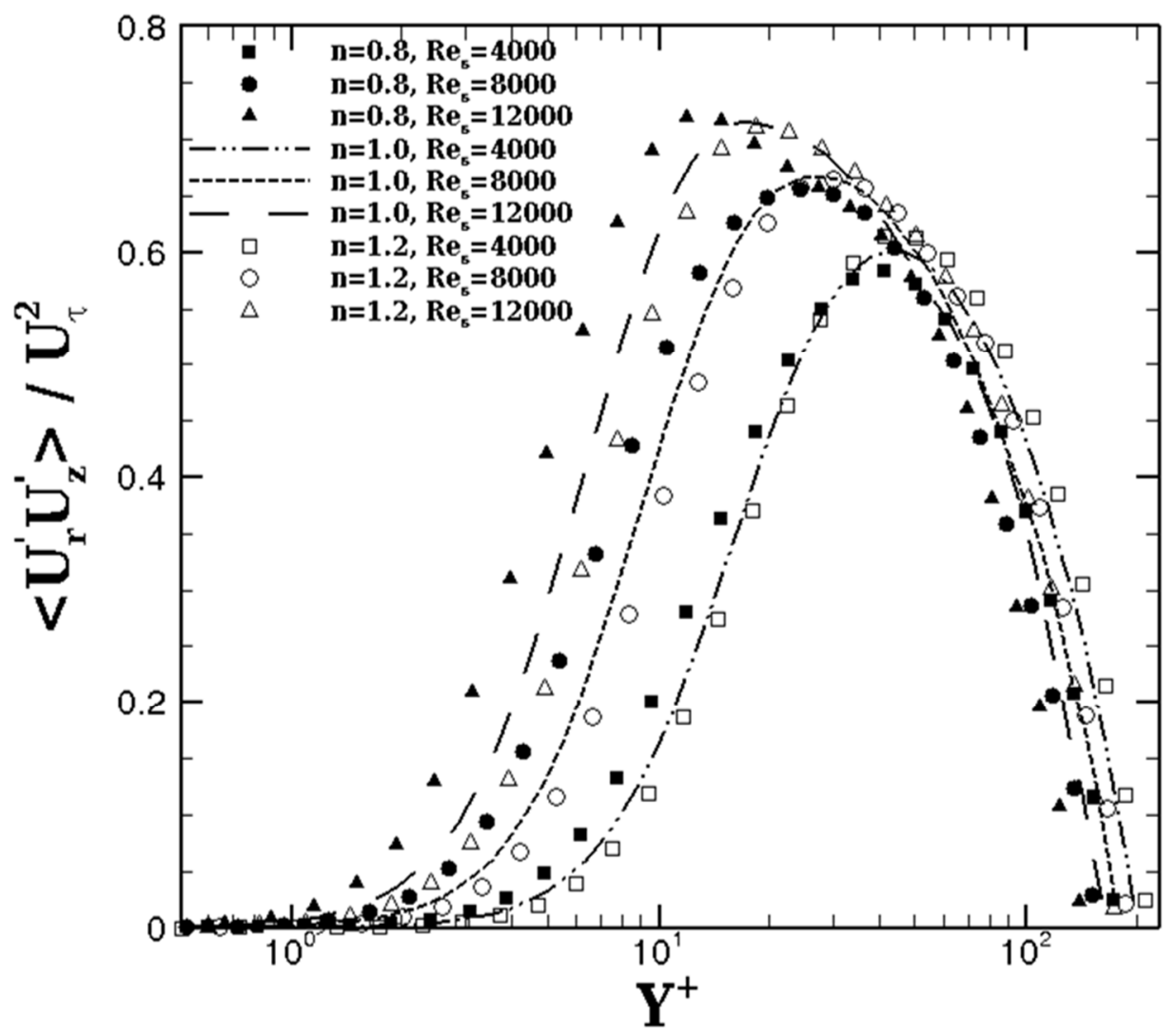

Fig.7b Effect of $\mathrm{Re}_{\mathrm{s}}$ on Reynolds shear stress. 


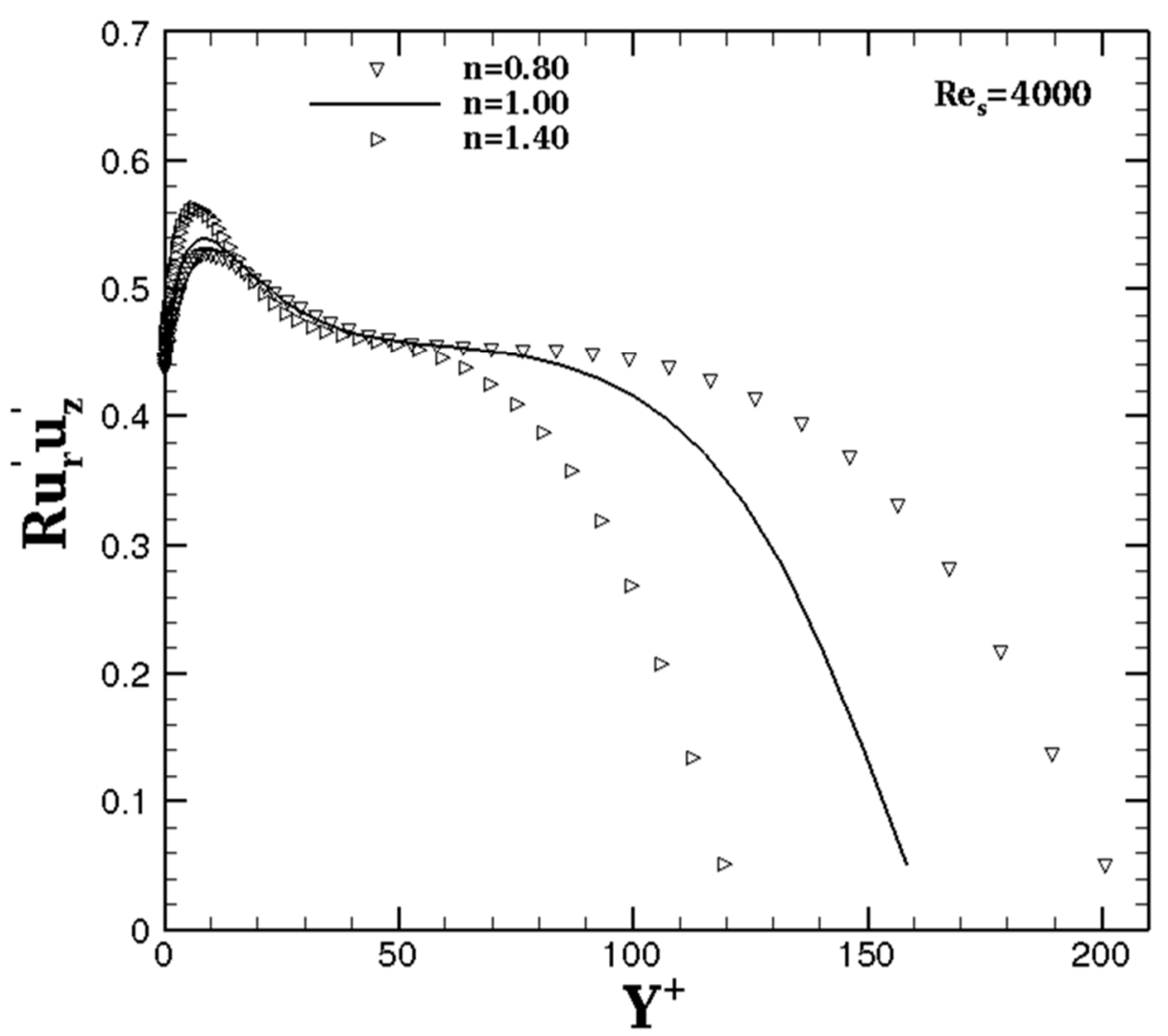

Fig.8 Cross-correlation coefficient.

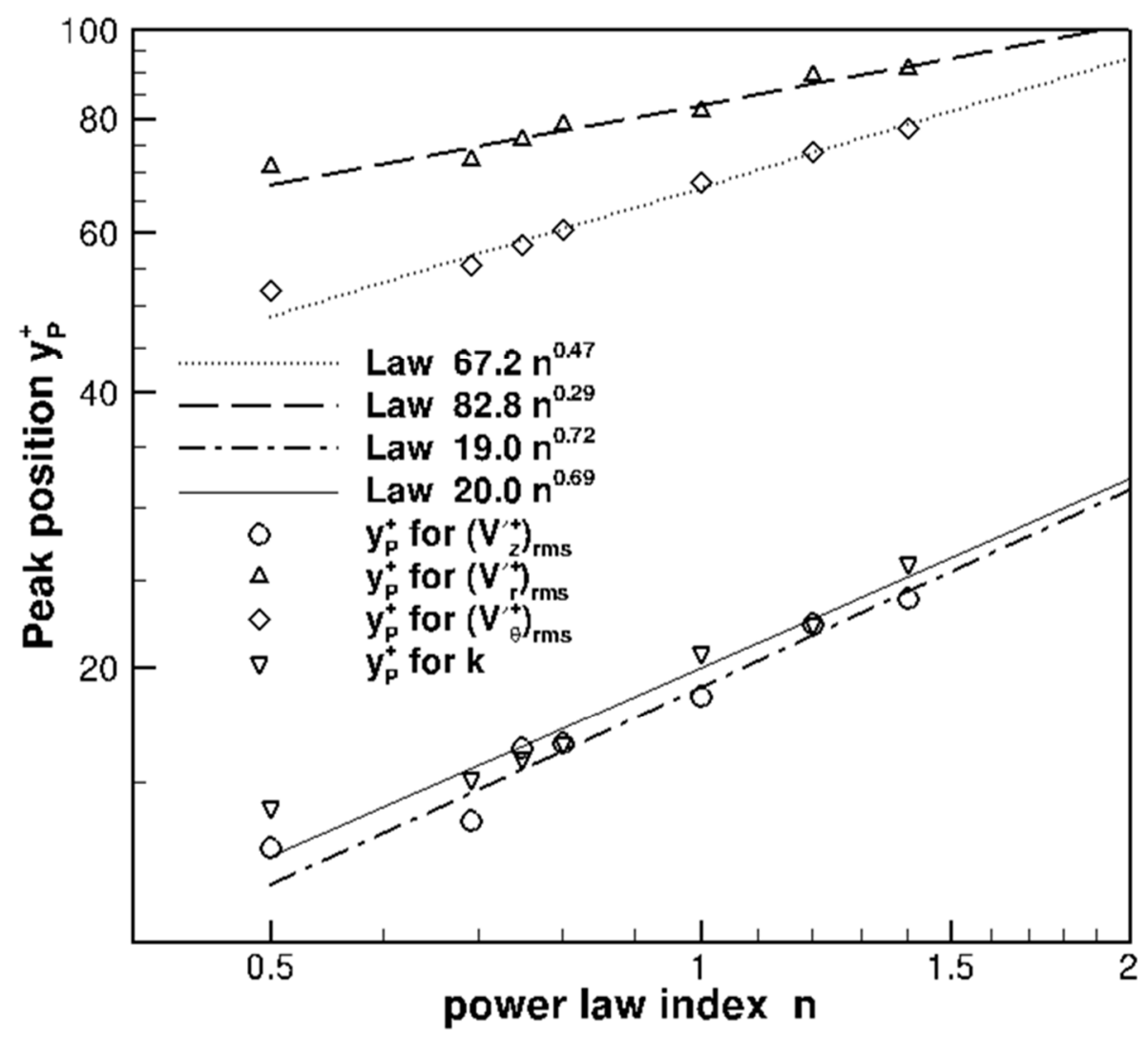

Fig.9 Peak position of RMS velocity fluctuations and kinetic energy. 


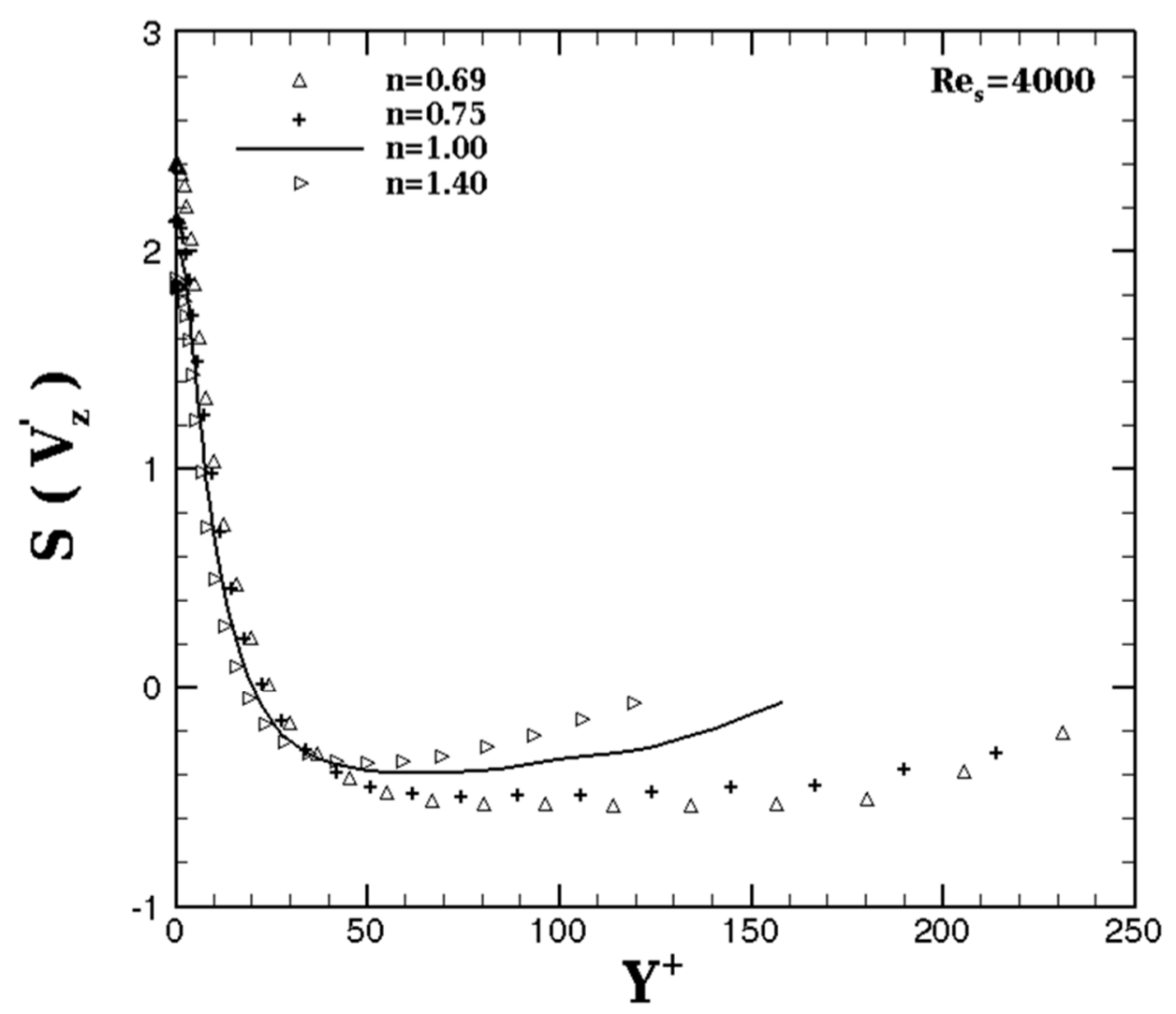

Fig.10a Skewness of axial velocity fluctuations: effect of $n$.

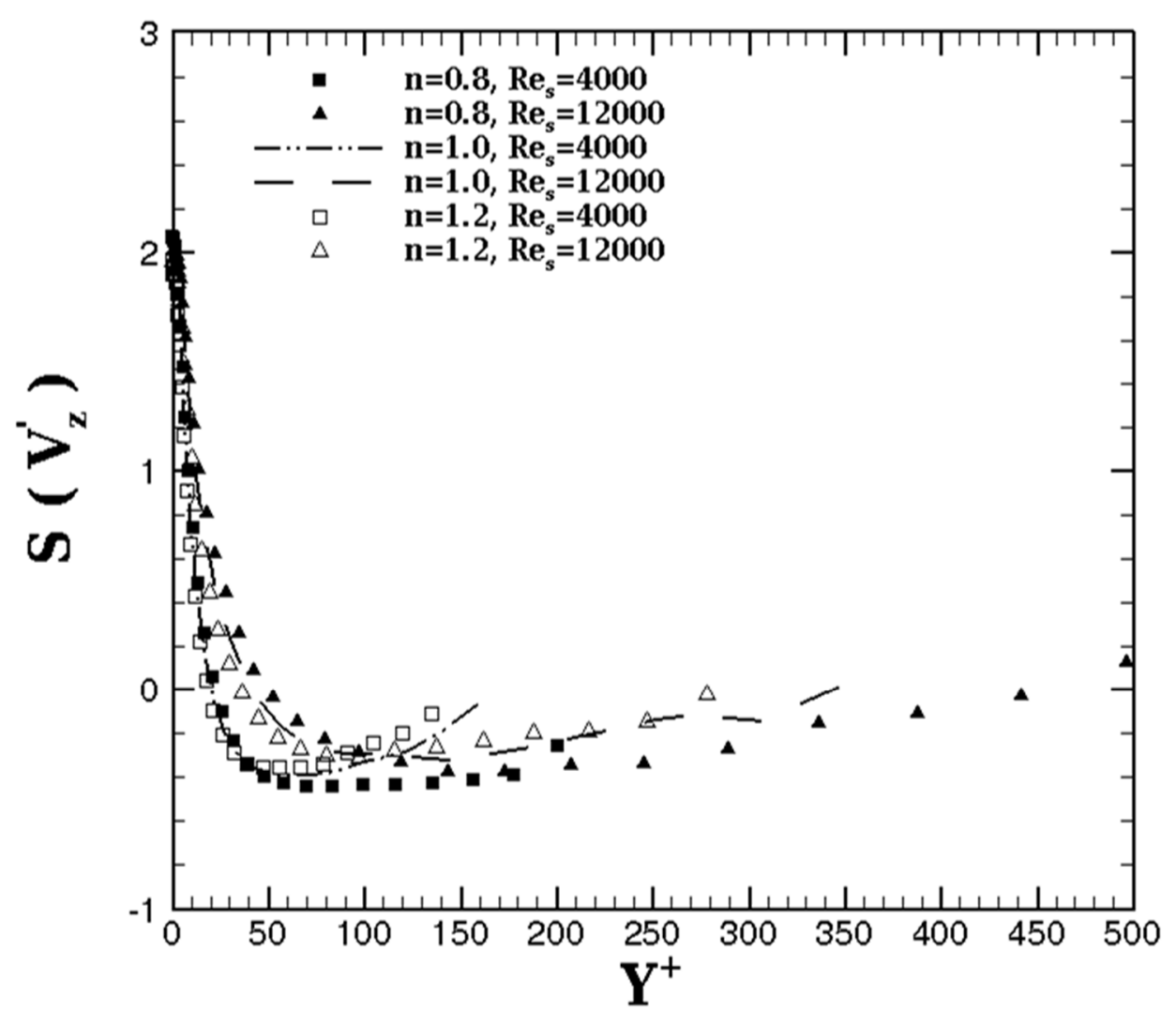

Fig.10b Skewness of axial velocity fluctuations: effect of $\mathrm{Re}_{\mathrm{s}}$. 


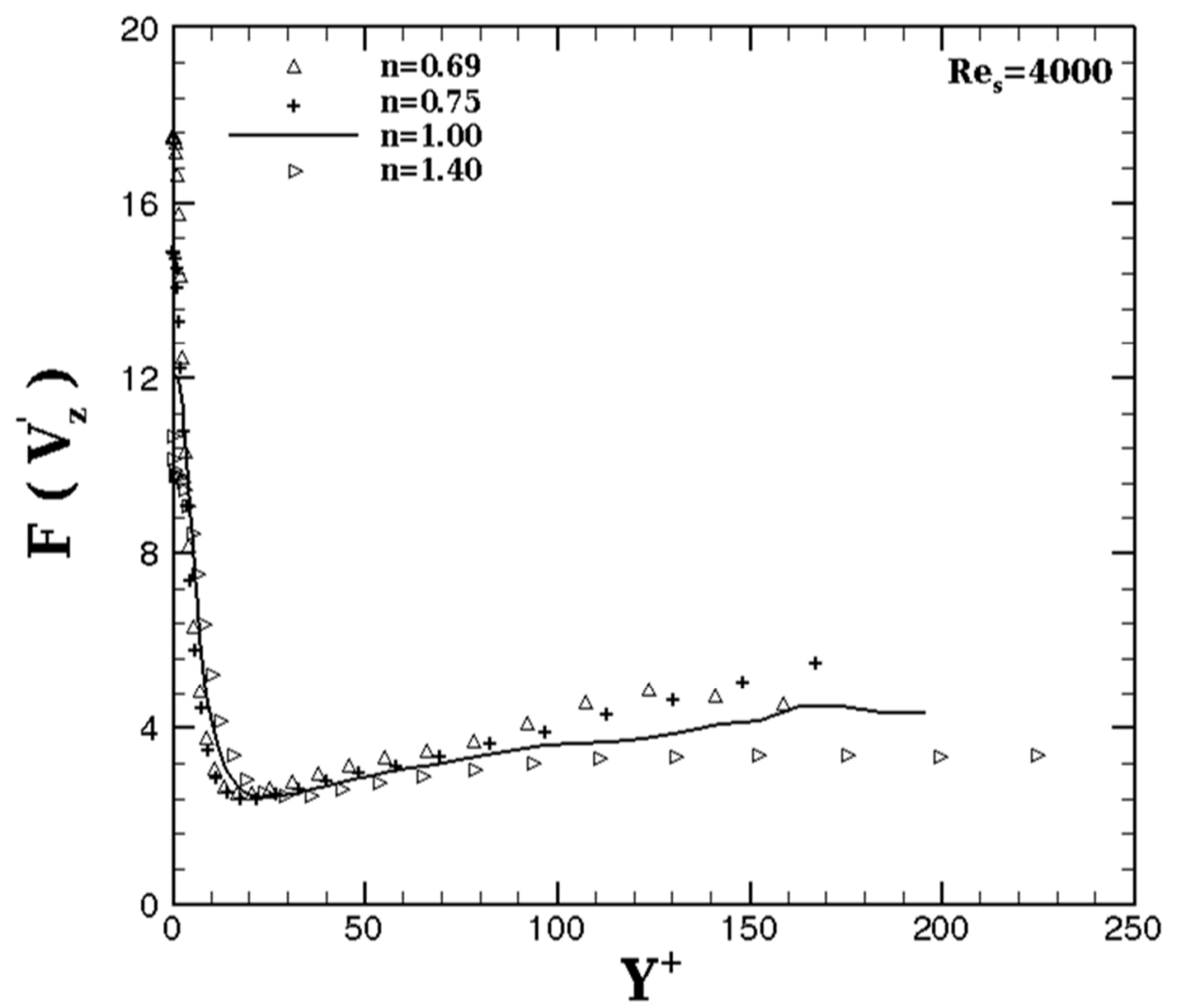

Fig.11a Flatness of axial velocity fluctuations: effect of $n$.

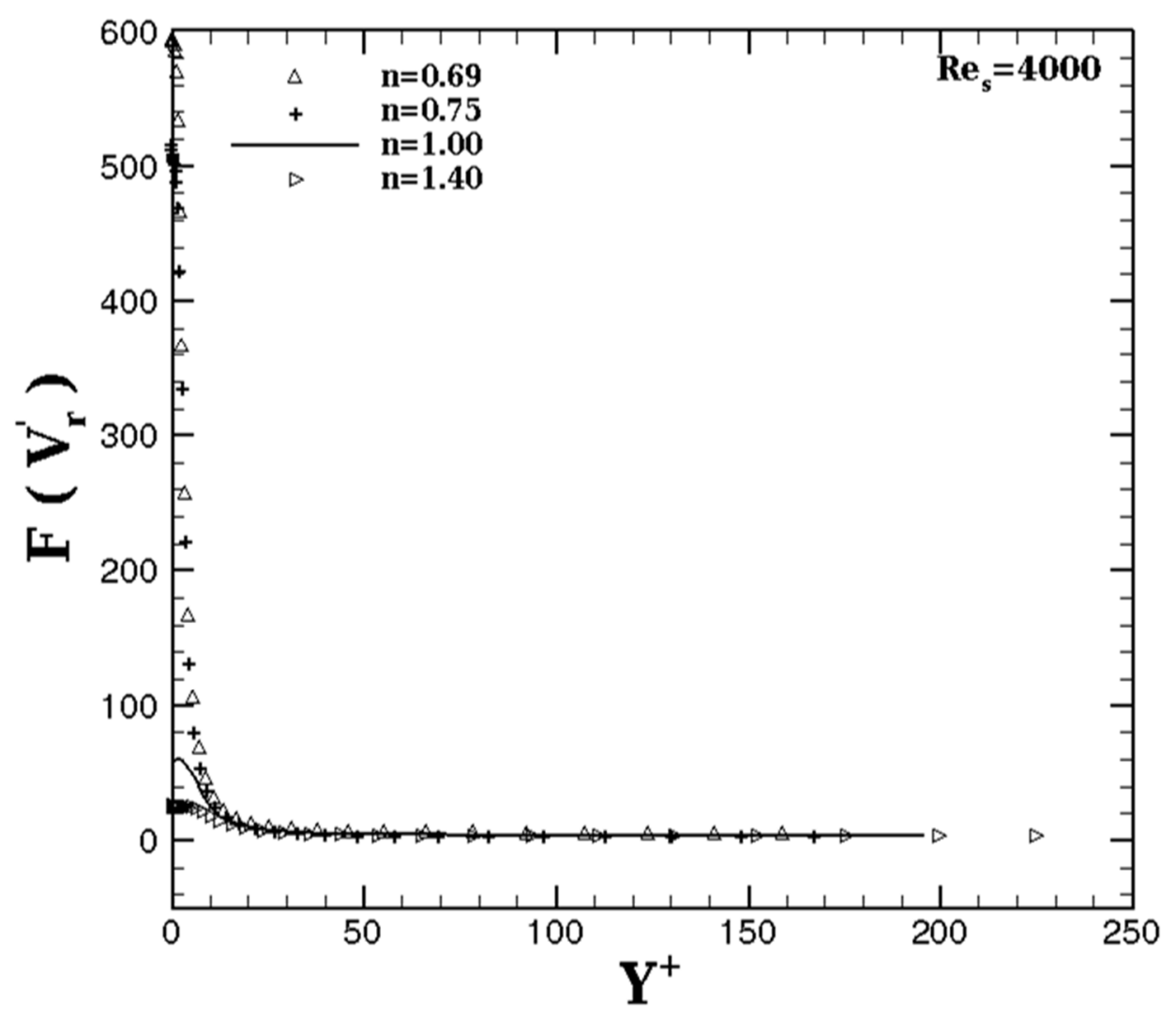

Fig.11b Flatness of radial velocity fluctuations: effect of $n$. 


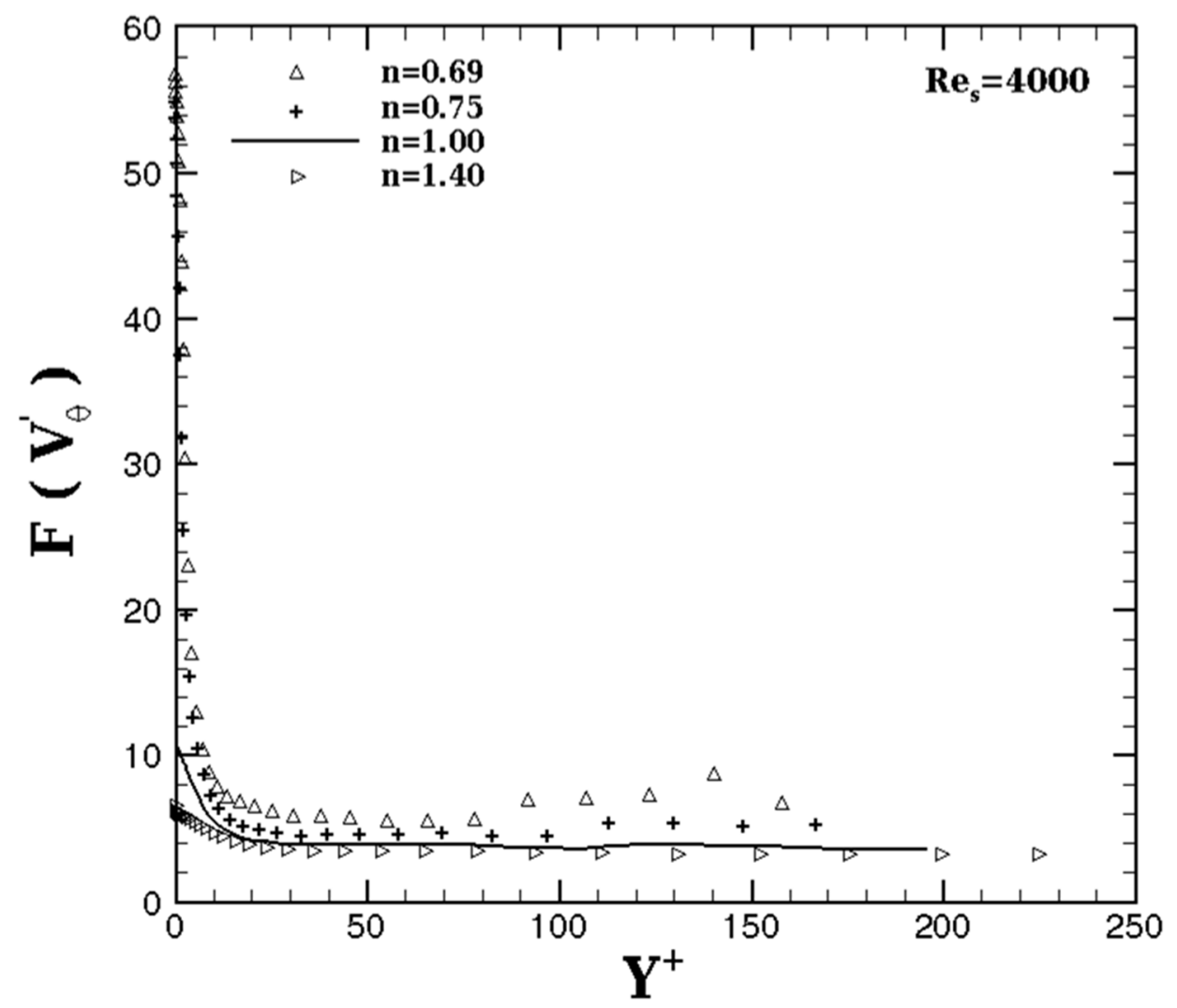

Fig.11c Flatness of azimuthal velocity fluctuations: effect of $n$.

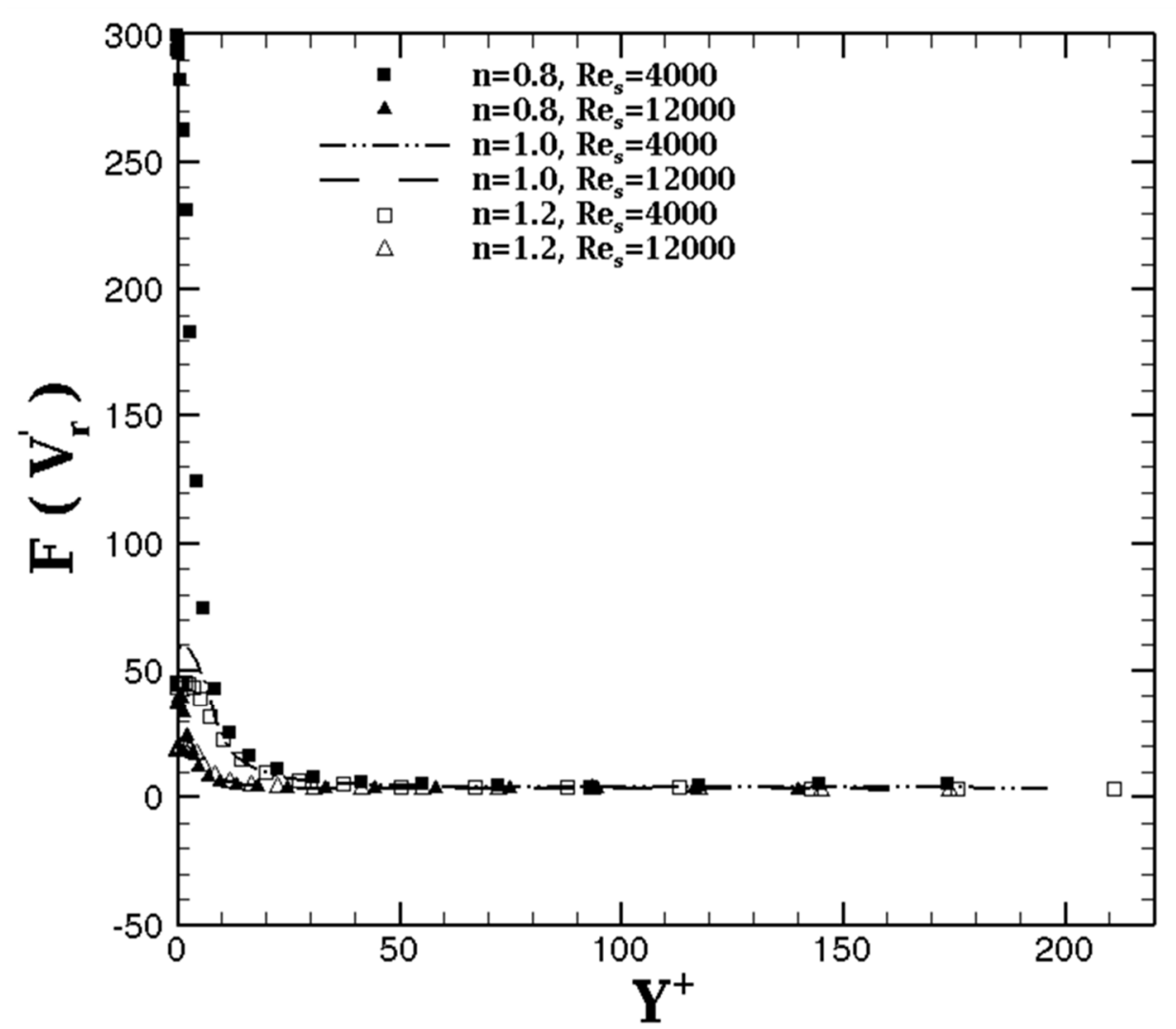

Fig.12 Flatness of radial velocity fluctuations: effect of $\operatorname{Re}_{\mathrm{s}}$. 


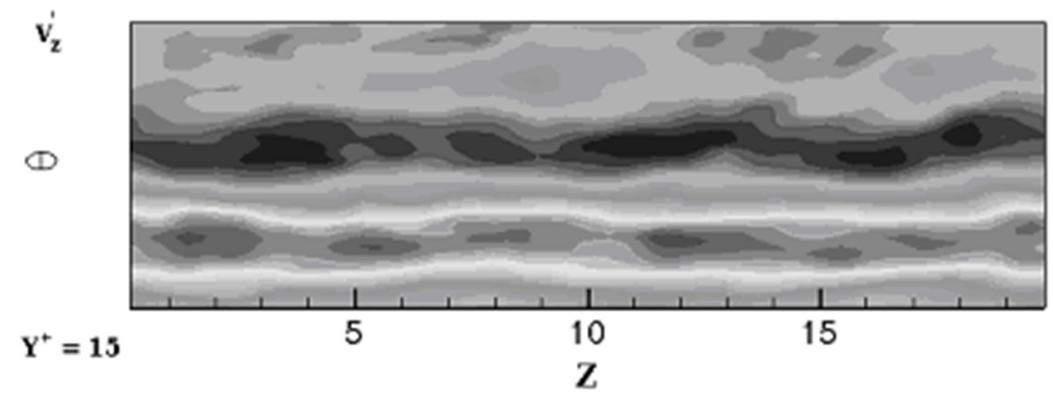

(a)

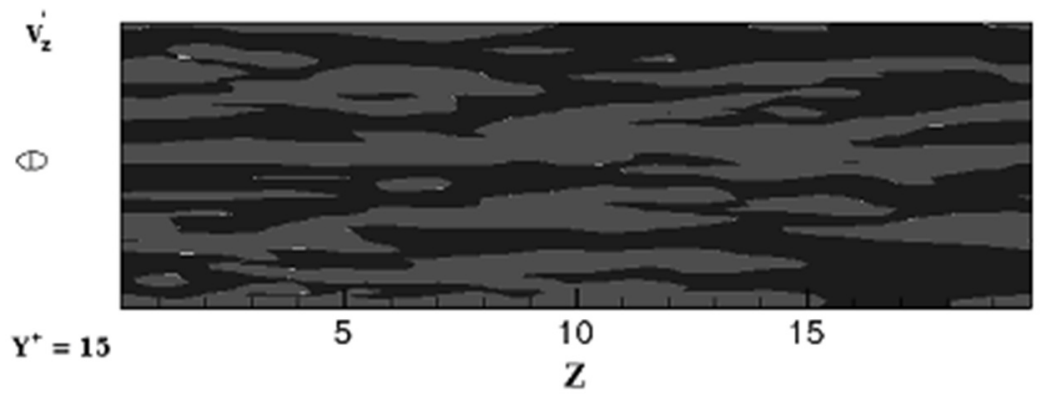

(b)

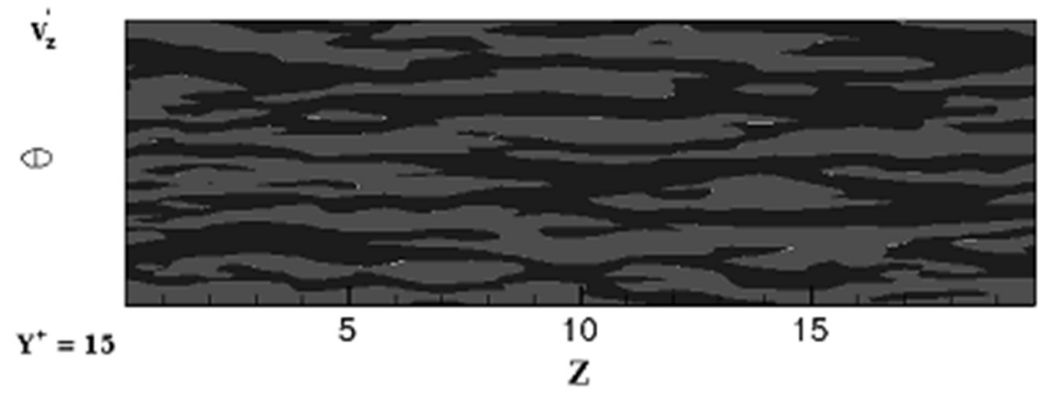

(c)

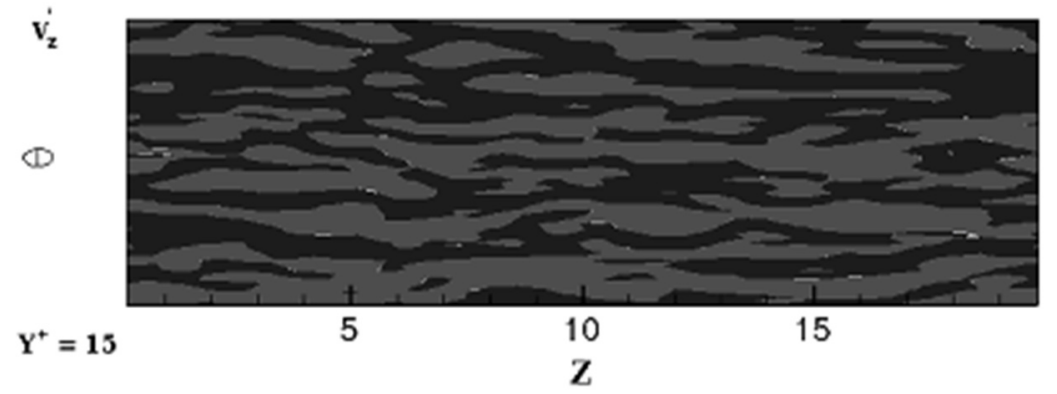

(d)

Fig. 13 Contours of resolved axial velocity field at $\mathrm{y}^{+} \sim 15$ : (a) $\mathrm{n}=0.5$, (b) $\mathrm{n}=0.75$, (c) $\mathrm{n}=1.0$, (d) $\mathrm{n}=1.2$. 

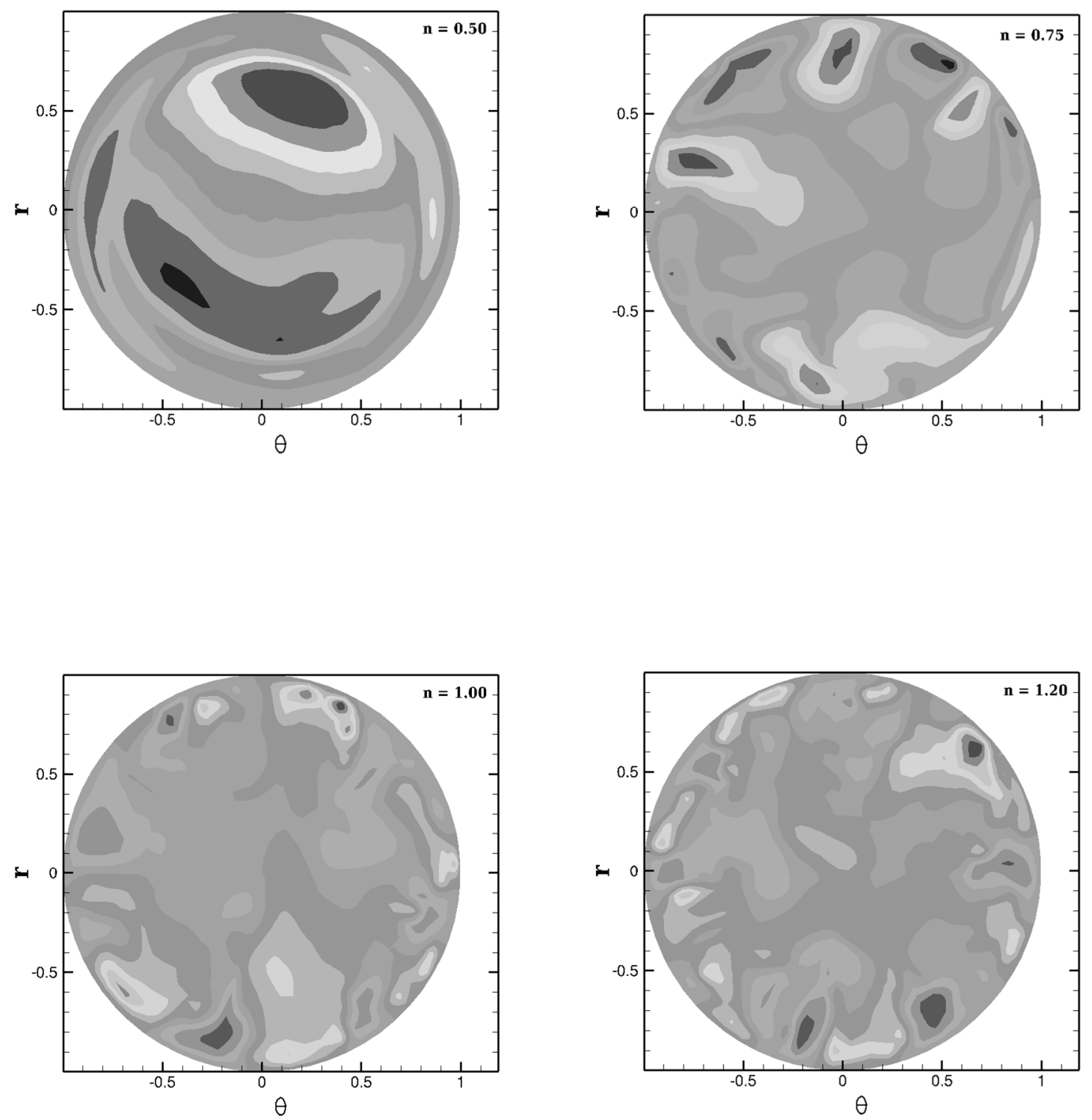

Fig. 14 Contours of resolved axial velocity field in (r- $\theta)$-plane:

(a) $\mathrm{n}=0.5$, (b) $\mathrm{n}=0.75$, (c) $\mathrm{n}=1.0$, (d) $\mathrm{n}=1.2$. 